\title{
Development of Raman micro-spectroscopy to characterize human ovarian cancer cells
}

\author{
by
}

Hamid Moradi

A thesis submitted to the Faculty of Graduate and Postdoctoral Affairs in partial fulfillment of the requirements for the degree of

\author{
Master of Applied Science \\ in \\ Biomedical Engineering
}

Ottawa-Carleton Institute for Biomedical Engineering

Department of Physics

Carleton University

Ottawa, Ontario

(C) 2016

Hamid Moradi 


\begin{abstract}
Raman spectroscopy is a vibrational spectroscopy technique and has demonstrated highly desirable unique analytical capabilities throughout the field of biomedical optics. It has the ability to obtain sensitive measurements of molecular composition, structure and dynamics from very small sample volumes in a non-destructive, non-invasive and label free manner. This makes it useful in the study of the cells as well as tissues. In this work a Raman micro-spectrometer system was developed and applied in vitro to discriminate between the ovarian carcinoma cell lines A2780s (parental wild type) and A2780cp (cisplatin cross radio-resistant variant). These two cell lines represent a good model of tumor tissues of similar origin but with different intrinsic chemo- and radio-sensitivities. Moreover, their radiobiological behavior has been extensively studied and their survival curves under different irradiation schemes are known. The Raman spectra collected from individual cells undergo initial preprocessing (background subtraction, normalization and noise reduction) to yield true Raman spectra representative of the cells. These spectra are analyzed with Principal Component Analysis (PCA) followed by Linear Discriminant Analysis (LDA) to yield a strong separation between the cell lines. The objective of this work was to characterize the spectral differences between the two cell types in order to determine the underlying biochemical basis for this separation. The multivariate classification model constructed using such Raman spectra of ovarian cancer cells could potentially be utilized for early prediction of tumor response.
\end{abstract}




\section{Acknowledgements}

This project provided many challenges, and I was fortunate to receive support from many great people along the way. First and foremost, I would like to thank my supervisor, Dr. Sangeeta Murugkar for her patience and guidance throughout this journey. It's been an absolute privilege working with her. Many thanks also to Carleton Biophotonics Research Group (CBRG) Abrar Ahmad and Dean Sheperdson for helping me with construction of our system, and without them I strongly doubt we would have been capable of getting it running in such short time. I would like to thank my dearest friend Nima Sherafati for helping me whenever I needed him. His support has been a guiding light for me. I would also like to thank our new members, Christopher Dedek, Harry Allen, and Achint Kumar, for their dedication in continuing this work. I wish them all the best with the project.

I would like to thank Dr. Barbara Vanderhyden and Nhung (Rose) Vong for providing the cell samples for this project. I would also like to thank Dr. Libni Eapen, Dr. Gosia Niedbala and in particular Dr. Balazs Nyiri for irradiating cells and shipping to our lab. Dr. Nyiri, I thank you for all your guidance and support, coming after hours and during holidays offering your help. I am deeply indebted to everyone in Dr. Ruth Willkin's group for all their financial support and assistance in Health Canada. In particular, I would like to thank Dr. Vinita Chauhan and Dr. Sami Qutob for helping us purchase the automated stage for our setup.

The Physics Department staff at Carleton University also deserve many thanks. Thanks to Mike Antunes and Penka Matanska for allowing us to borrow their equipment 
and provide us with their expertise when we needed most. Thank you also to Philippe Gravelle in the Physics machine shop for your invaluable help.

Last but not least, I would like to thank my family for their immeasurable support throughout my graduate studies. A particular thank you to my wife, Alesya, for working so hard to provide me time and resources necessary to complete this project and being a great mother to our children. Special thanks to my Mom and my siblings for their endless love and support. 


\section{Statement of Originality}

The author, Hamid Moradi, hereby certifies that all work which went into producing this thesis was his own. The authors of any other ideas or concepts which were used have been acknowledged and referenced. Assembly and data acquisition were performed by the author under the guidance of his supervisor, Dr. Sangeeta Murugkar.

The author would also like to note that permission has been obtained for the images which were reproduced in this thesis and their sources are fully acknowledged in accordance with the standard referencing practices. The cells were grown and fixed by N.H. Vuong (Rose) in Dr. Vanderhyden's lab at The Ottawa Cancer Center and transported to our lab at Carleton University. 


\section{Table of Contents}

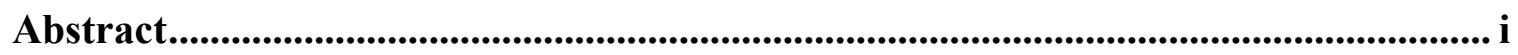

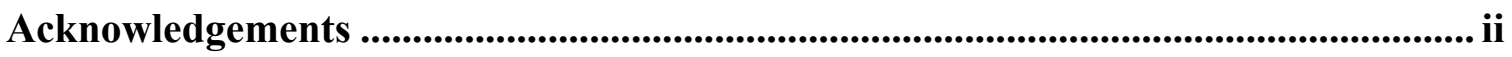

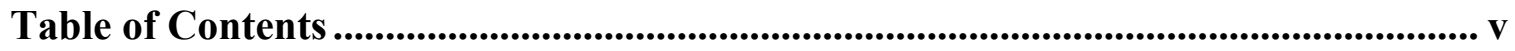

List of Tables .............................................................................................................................. viii

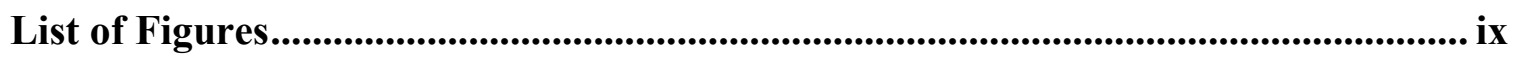

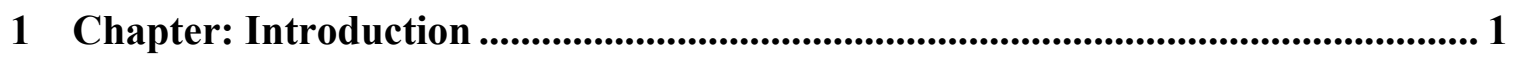

1.1 Overview of Modern Biomedical Raman Spectroscopy ............................................. 1

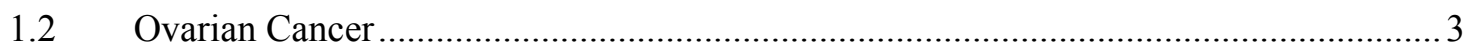

1.3 Raman Spectroscopy and Ovarian Cancer ......................................................... 4

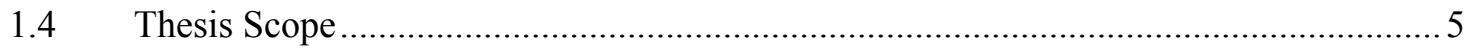

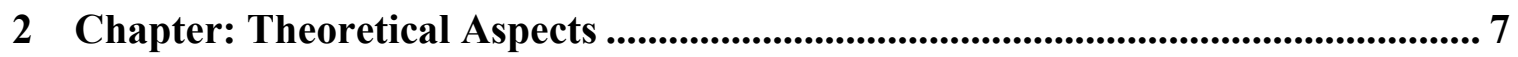

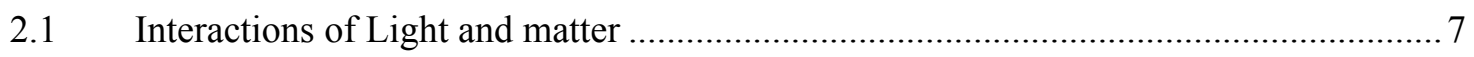

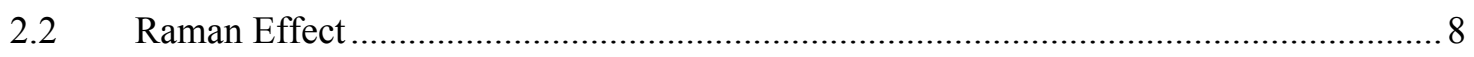

2.3 Classical Description of Raman Scattering ......................................................... 9

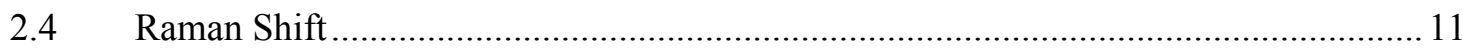

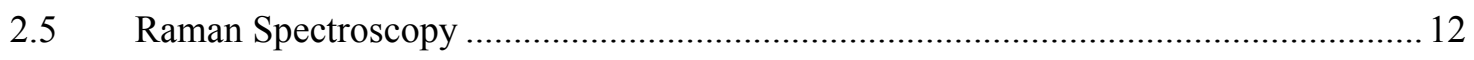

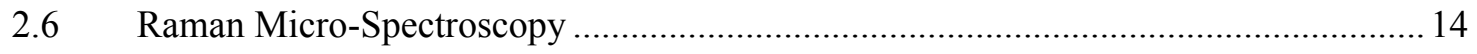

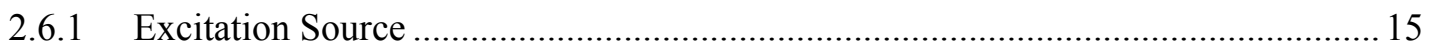

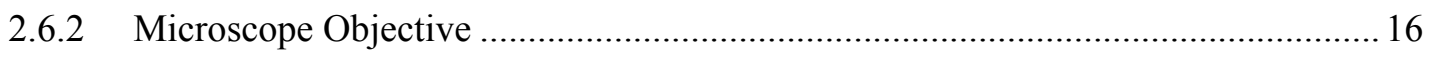

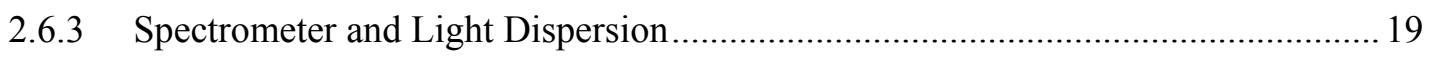

2.6.4 Charge-Coupled Devices and Light Detection...................................................... 20

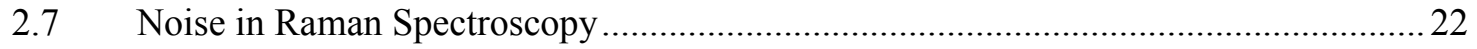

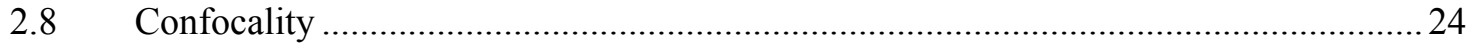




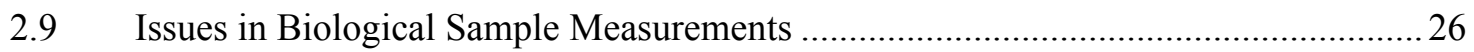

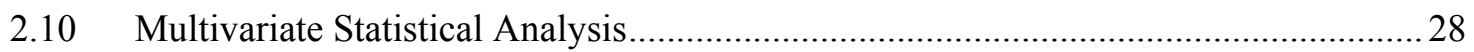

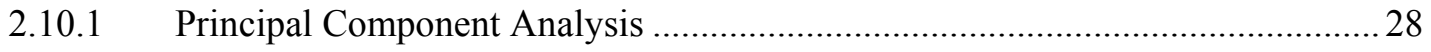

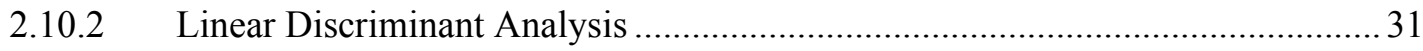

3 Chapter: Instrument Development and Performance................................................ 33

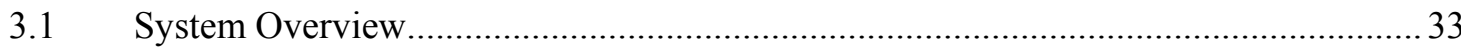

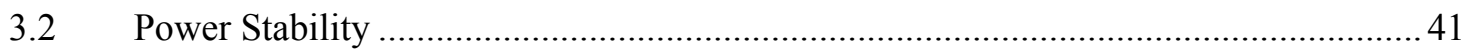

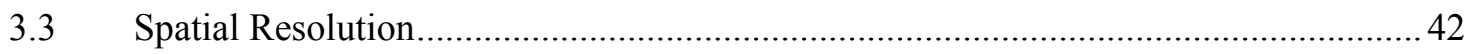

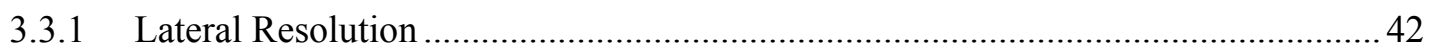

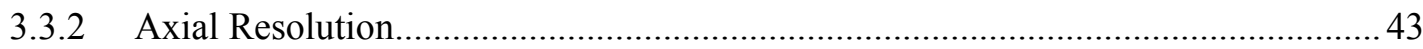

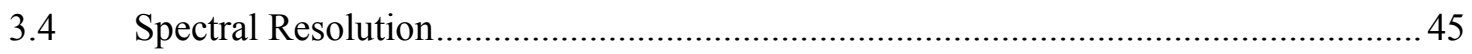

4 Chapter: Materials and Methods .............................................................................. 48

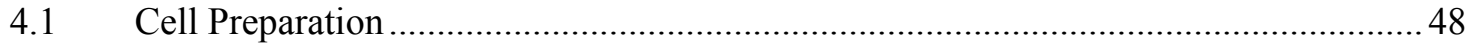

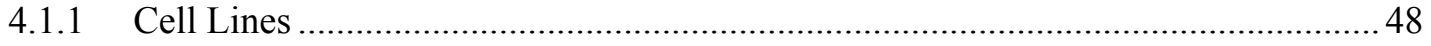

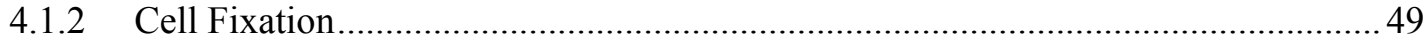

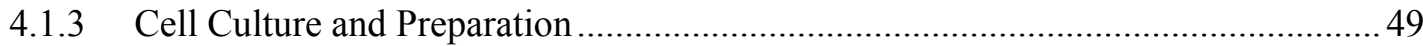

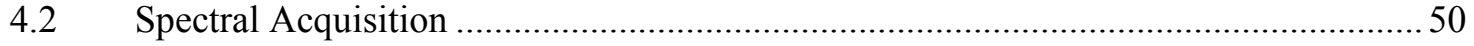

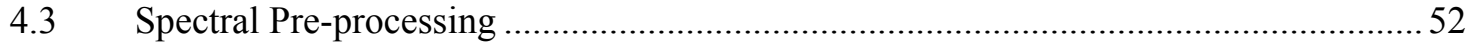

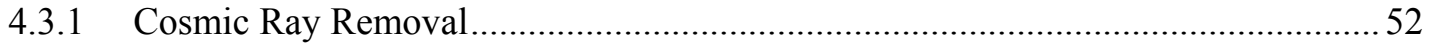

4.3.2 Background Subtraction/Baseline Removal......................................................... 53

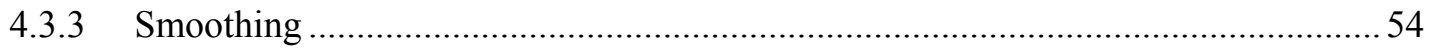

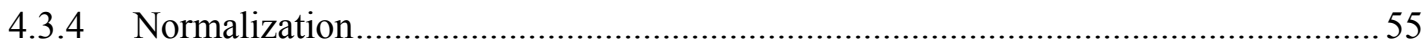

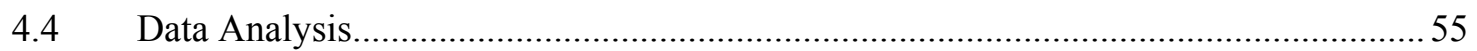

5 Chapter: Results and Discussion .............................................................. 57

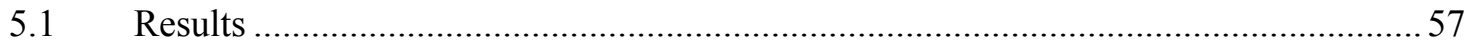




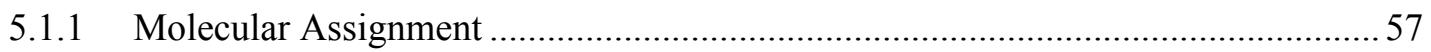

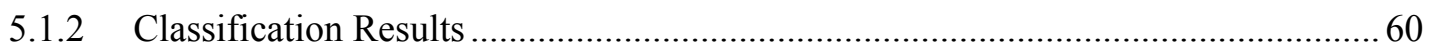

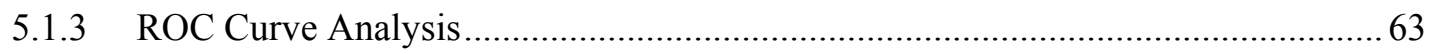

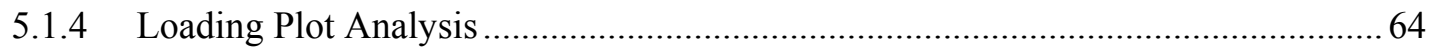

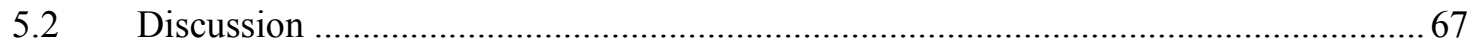

6 Chapter: Conclusion and Future work .............................................................. 70

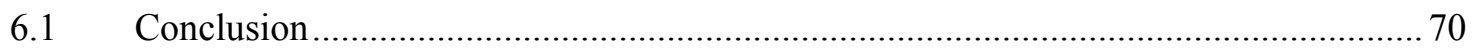

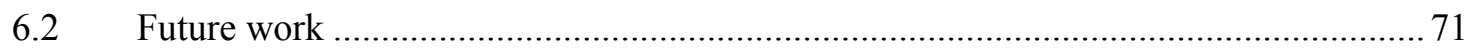

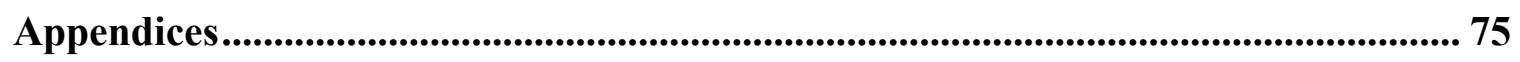

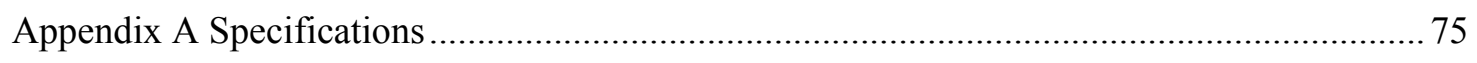

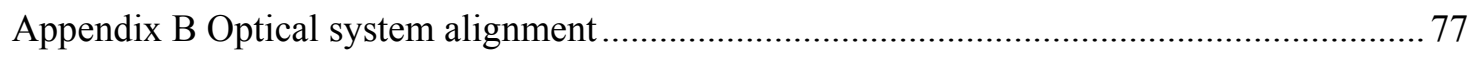

Appendix C Instrument Calibration and Spectrum Acquisition ........................................... 79

Appendix D Algorithm for Cosmic Background Removal and Spectral Acqusation ............... 81

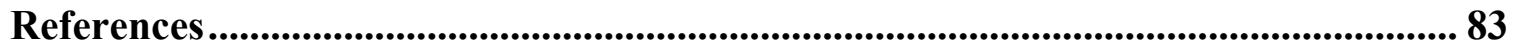




\section{List of Tables}

Table 5.1: Main peaks observed in the Raman spectra of fixed A2780 cells and molecular assignments according to Refs [48-53]. Abbreviations: (d) nucleic acid, (c) carbohydrate, (p) protein, (1) lipid

Table 5.2: Confusion matrix for the test data classification and for LOOCV obtained from PCA-LDA of A2780cp and A2780s cells.

Table 5.3: Tentative Raman band assignments for LD Loading (Figure 5) tabulating major peaks based on Refs [48-53]. Abbreviations: (d) nucleic acid, (c) carbohydrate, (p)

protein, (1) lipid 66 


\section{List of Figures}

Figure 2.1: Energy level diagram of possible scattering interactions between incident photon and a molecule: a) elastic (Rayleigh), b) Stokes and c) Anti-Stokes Scattering .... 9

Figure 2.2: Basic schematic of the typical Raman spectroscopy system......................... 13

Figure 2.3: Basic schematic of the typical Raman micro-spectroscopy system ............... 14

Figure 2.4: Illustration of the microscope objective with light cone. The angle $\boldsymbol{\theta}$ is one half of the angle subtended by the cone. The dimensions of the laser spot are indicated by

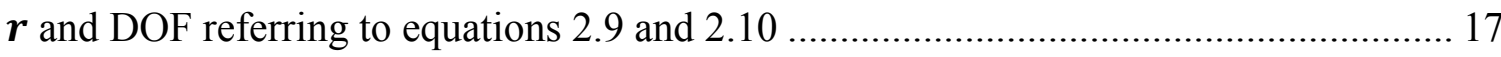

Figure 2.5: Schematic description of Czerny -Turner design for a spectrometer [www.azooptics.com/Article.aspx?ArticleID=309] 19

Figure 2.6: Quantum efficiency curves for back-illuminated (BI), back-illuminated deepdepletion (DD) and front-illuminated (FI) CCD detectors as a function of wavelength $[21]$

Figure 2.7: A diagram showing the pinhole function. The green dot is the light coming from focal plane and the red and yellow are the signal coming from out of focus planes [adapted from 26]

Figure 2.8: Comparison of spectra produced by $785 \mathrm{~nm}$ for different substrate materials $[29]$

Figure 2.9: Schematic representation of axes found using PCA and LDA for two class data [adapted from 33]

Figure 3.1: Custom confocal microscope coupled to a Raman spectrometer. (1) $785 \mathrm{~nm}$ laser (2) Optics for laser beam collimation and expansion (3) Dichroic mirror (4) Microscope Objective (5) Automated x-y-z stage (6) CCD camera for bright-field 
imaging (7) Laser rejection filter (8) Pinhole lens (9) Pinhole (10) Focusing optics and (11) Spectrometer and CCD detector. 33

Figure 3.2: Transmission efficiency curve for dichroic beam splitter [www.semrock.com] 35

Figure 3.3: Transmission efficiency curve for Long-pass filter [www.iridian-opticalfilters.com] 36

Figure 3.4: Optical coupling NIR achromatic doublet pair's schematic

[www.thorlabs.com] 38

Figure 3.5: Shamrock SR-303i with Czerny Turner configuration [www.andor.com] ... 39

Figure 3.6: Quantum efficiency of CCD detector used in this system. [www.andor.com]

Figure 3.7: Power stability measurement for the laser 41

Figure 3.8: Experimental axial responses for a thick polystyrene sheet sample 44

Figure 3.9: The derivative of the detected intensity function shown in figure 3.8 for thick polystyrene sheet sample 45

Figure 3.10: Spectral peak at $1001.4 \mathrm{~cm}^{-1}$ of polystyrene bead and fit to calculate FWHM to measure the spectral resolution of the system.

Figure 4.1: Bright field image of fixed ovarian cancer cell with the bright white spot

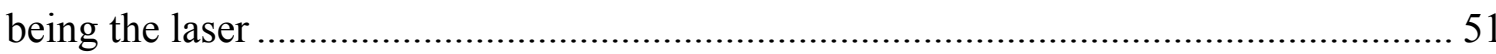

Figure 4.2: Silicon peak used as a system check ................................................. 52

Figure 5.1: Mean Raman Spectrum of A2780CP (red) and A2780S (blue) cells 57

Figure 5.2: The empirical and fitted cumulative distribution function of the A2780s and A2780cp cells in the training set versus the LDA score. 61 
Figure 5.3: The histogram for the LDA scores of the A2780s and A2780cp cells in the training set along with the fitted probability density function derived from the normal distribution fits in figure (5.2), and at the bottom is the histogram of LDA scores for the test data for A2780s and A2780cp cells

Figure 5.4: Receiver Operator Characteristics for LOOCV models (empirical and fit) of

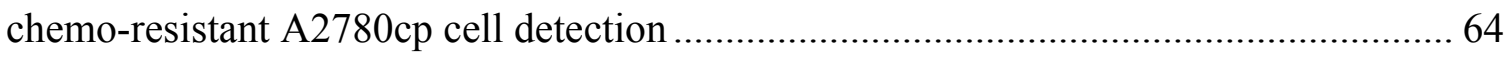

Figure 5.5: Mean LD Loading with important Raman bands indicated by red dots ....... 65 


\section{Chapter: Introduction}

\subsection{Overview of Modern Biomedical Raman Spectroscopy}

Raman spectroscopy is a very versatile optical technique that has been recognized as a powerful tool for bioanalytical and biomedical applications. It allows noninvasive acquisition to obtain molecular information with high spatial sub-cellular resolution. Raman spectroscopy measures the transitions between vibrational levels of the molecule, and relies on the inelastic scattering of incident radiation by the molecule. Raman spectra contain information on vibrations that provide a highly specific fingerprint of the molecular structure and biochemical composition of cells and tissue. This technique is sensitive to biochemical differences and may be used in the discrimination of cell types or in monitoring the progression of disease. However, differences between cell types are minimal and often multivariate analysis is needed to tease out small variations.

Another method of vibrational spectroscopy is infrared (IR) spectroscopy, which operates by exciting molecular vibrations via absorptions in the mid-infrared $(2.5-50 \mu \mathrm{m}$ wavelength) range. However, water molecules in this range produce a strong spectrum that often hides a lot of the biomolecular information and can make the analysis of substances in an aqueous environment difficult. In contrast, Raman spectroscopy in general can be performed in the ultraviolet, visible and near infrared spectral range (200$1064 \mathrm{~nm}$ ), where Raman scattering of the water molecules is much weaker. However, near infrared lasers are used for studying biological samples to avoid any degradation and photodamage. Moreover, the preparation of samples for IR spectroscopy is often more complex than for Raman spectroscopy and usually is restricted to thin, dehydrated samples [1]. Finally, Raman spectroscopy typically uses a laser wavelength of $532 \mathrm{~nm}$ or 
$785 \mathrm{~nm}$, so that a spatial resolution of approximately half the wavelength $(250-350 \mathrm{~nm})$ can be achieved.

In fact, the sub-cellular resolution achieved is similar to that achieved in fluorescence imaging and is much better than the resolution achieved by medical diagnostic techniques such as ultrasound, Magnetic Resonance Imaging, and Positron Emission Tomography. An additional benefit of Raman spectroscopy is that it is noninvasive [2]. This property plays an important role in combination with microscopy. Therefore, Raman spectroscopy has a potential for cell and tissue diagnosis, particularly in the area of in vivo applications [1].

Recent advances in Raman spectroscopy have generated a surge of interest in biomedical applications, particularly in the field of oncology $[3,4]$. Raman spectroscopy can thus provide real-time, noninvasive or minimally invasive, differential diagnosis of cancer. A large number of studies have been performed on tissues and cells, often comparing healthy with cancerous such as blood cells [3], breast [4], cervical [5], etc.

Raman spectroscopy is also suitable for use with fiber-optic probes, making it an ideal medical diagnostic tool in cancer research and clinical setting. Fiber-optic probes designed for in vivo use must overcome signal-to-noise ratio challenges, and unwanted Raman signals and photoluminescence generated in the laser delivery fibers. For certain applications, all this must be achieved with a small probe to enable access to body cavities, and in a sufficiently short time to allow accurate measurement from a moving target organ [3]. Recently handheld fiber-based probes have been developed for spectroscopic assessment of brain, breast, oral, and skin cancers and they are currently on 
clinical trials. However a greater body of evidence is necessary before many of these fiber-coupled probes are fully validated for clinical use.

Raman spectroscopy also requires the implementation of advanced statistical techniques for rapid, accurate classification and discrimination between normal and cancerous cells and tissues. This combination could greatly reduce operating time and offer more reliable information than conventional histopathological methods and improve the clinical outcome for the patient. However at present, performing Raman spectroscopy with advanced statistical analysis requires input from multidisciplinary experts, careful interpretation of the data and stringent validation before it can fulfill its promise [4].

\subsection{Ovarian Cancer}

Ovarian cancer has the highest mortality of all gynecological cancers. Overall, it is the fifth leading cause of death among women in North America [6]. The underlying biology of the disease is still not well understood. The disease develops with few symptoms and it is estimated that only $20 \%$ of the disease is detected before tumor metastases. There is no effective screening method, resulting in late-stage diagnosis and high ( $>60 \%)$ mortality rate. The disease normally presents itself after wide-spread dissemination and the standard treatment is aggressive surgery followed by combined carboplatin and taxol chemotherapy. Most of the patients initially respond to chemotherapy, however more than $75 \%$ of patients undergo relapse after treatment and $\sim 30 \%$ of patients fail to respond and/or progress rapidly within 1 year of treatment.

The root cause of this resistance is attributed to the existence of a small population of platinum-resistant cells in the ovarian tumour before treatment [7]. After 
treatment, the platinum-sensitive cells are destroyed while the platinum-resistant cells multiply, resulting in a platinum-resistant tumour. Therefore, the primary goal at the time of surgery is to remove all visible and microscopic metastasized tumour. The ability to resect platinum-resistant disease in particular, will increase the chance of achieving a cure.

Several methods to screen for treatment resistance have been reported, including treatment of freshly isolated and cultured tumor cells [8], detection of biomarkers [9], and use of positron emission tomography [10]. None of these tests have been adopted into routine clinical practice, in part due to the need for further investigation, and they can be expensive and at present, do not facilitate discrimination at the cellular level and in real time.

Positive significant impact in the cure of ovarian cancer lies in the development of better diagnostic tools than are currently available. Hence our interest in investigating optical spectroscopic techniques and well-tailored multivariate statistical analysis to distinguish between platinum-sensitive and platinum-resistant ovarian tumor cells, could enhance current diagnostic methods.

\subsection{Raman Spectroscopy and Ovarian Cancer}

Considering the prevalence of ovarian cancer and the enthusiastic use of Raman spectroscopy in so many oncological application, it is surprising that so few Ramanbased studies have been conducted into the disease [11]. The Krishna group has found significant differences between spectral profiles of normal and malignant fresh and fixed ovarian tissues [12,13]. The Martin group has characterized alterations in the 
biomolecular signatures of human blood plasma/serum obtained from ovarian cancer patients using Raman spectroscopy [14]. Our group have recently shown that Raman micro-spectroscopy is a highly sensitive technique to discriminate between normal and malignant mouse ovarian epithelial cells in vitro [15].

While these studies have focused on the early detection of ovarian cancer, there seems to be a lack of published work on the detection of chemo-resistance in ovarian cancer, and discrimination between sensitive and resistant cancers.

\subsection{Thesis Scope}

The primary goal of this work is twofold: (1) to design, develop, and characterize a Confocal Raman micro-spectroscopy system that is capable of investigating the structure and spectra of samples like human cells, and (2) to develop non-invasive, label free Raman spectroscopic discrimination of the fixed ovarian carcinoma cell lines that are inherently resistant or sensitive to chemotherapy. The type of microscope setup used in this work provides a spatial resolution that allows for collecting spectra from the A2780s (parental wild type) and A2780cp (cisplatin cross radio-resistant variant) cell lines, which have been provided by the Dr.Vanderhyden group. The spectra obtained from these cell lines have been used to construct a classification model in order to predict the species of future cell samples.

This thesis is divided into six chapters. Chapter 2 discusses the basic theoretical principles of Raman scattering and instrumentation in the Raman spectrometer system. The practical aspects related to the design of a confocal Raman micro-spectroscopy system are presented in Chapter 3, along with experimental characterization of its 
performance. Chapter 4 details the materials and methods applied to carry out the work presented in this thesis. Chapter 5 presents experimental results obtained by Raman micro-spectroscopy for molecular analysis of ovarian carcinoma cell lines A2780s and A2780cp cells.

Finally, future work and the conclusion are presented in Chapter 6 of this thesis. In this chapter, we also highlight the main conclusions drawn from the results presented in chapter 5 and the implications of these results. 


\section{Chapter: Theoretical Aspects}

Raman spectroscopy is an optical technique based on the interaction of light with the vibrational energy levels of molecules and can provide chemical fingerprints of cells, tissues or biofluids. Raman spectroscopy has been shown to be a powerful technique in the study of biological materials. In this chapter, the theoretical background of Raman spectroscopy and Raman micro-spectroscopy is introduced. The chapter will begin with a theoretical description of the Raman effect from the classical view. The theoretical aspects relevant to the main components of the Raman micro-spectroscopy system and its design consideration are then described. The last part of the chapter describes the mathematical methods used in the analysis of collected Raman data.

\subsection{Interactions of Light and matter}

When light interacts with matter, a variety of processes can occur. The photons may be scattered, absorbed, or transmitted. The absorption of photons leads to an excited state of the matter, which occurs when the photon energy is equal to the energy gap between the ground state of the molecule and the excited state. The excited molecule will return to the ground state by a radiative process such as fluorescence or non-radiative processes. It is this change which is measured in absorption spectroscopy.

It is also possible for a photon to interact with the molecule and scatter from it. In this case, the photon energy does not have to equal the difference between two energy levels of the molecule. If there is no change in photon energy, the process is called elastic scattering (Rayleigh scattering). This could be considered as an absorption of the photon energy and transfer the molecule to a virtual state, followed by an immediate de- 
excitation into the ground state, with re-emission of the photon. If the energy of the emitted photon is different from the incident photon this is called inelastic scattering, and we discuss this in the next section. We note that elastic scattering is the dominant scattering process, and only a small portion of light $\left(\sim 10^{-8}\right.$ of the incident beam) will scatter inelastically.

\subsection{Raman Effect}

The basic theory of Raman spectroscopy comes from the principle of inelastic scattering. It was first observed experimentally in 1928 by C.V. Raman and K. S. Krishnan [16]. Inelastic scattering is a phenomenon with an energy exchange between an incident photon and a molecule. Such interactions are also referred to as the Raman effect and exist in two forms. If the incident photon transfers energy to the molecule, the scattered photon will have a lower energy than the incident photon and will be scattered with a red shifted wavelength. This is known as Stokes Raman Scattering. Alternatively, if the photon interacts with a molecule initially in an excited vibrational state, the molecule may transfer the energy to the scattered photon with blue shifted wavelength, resulting in anti-Stokes Raman scattering. Stokes and anti-Stokes shift are symmetric with respect to the wavelength of irradiated light but the probabilities of their occurrence are different. At room temperature the anti-Stokes process occurs less often than the Stokes for most biological molecules, since most of the molecules are present in the ground vibrational state [17]. A schematic overview of the elastic and inelastic scattering is given in figure

\section{1.}




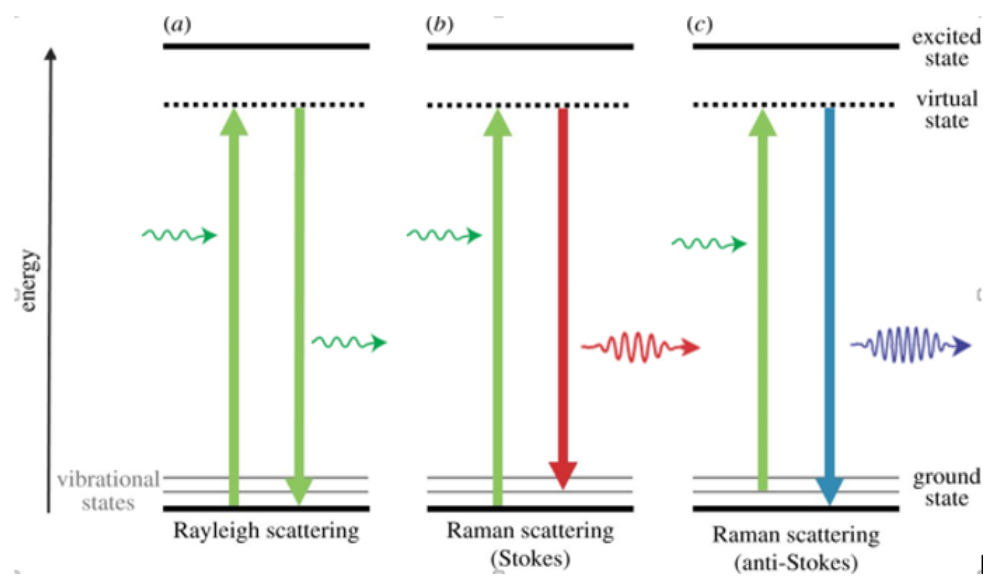

Figure 2.1: Energy level diagram of possible scattering interactions between incident photon and a molecule: a) elastic (Rayleigh), b) Stokes and c) Anti-Stokes Scattering

\subsection{Classical Description of Raman Scattering}

Classical interpretation of Raman scattering can be explained by the interaction of light incident on a molecule, which is viewed as an oscillating electric field $\boldsymbol{E}$ that induces an electric dipole moment $\boldsymbol{P}[18]$ :

$$
\boldsymbol{P}=\alpha \boldsymbol{E}=\alpha E_{0} \cos \left(2 \pi v_{0} t\right)
$$

where $\alpha$ is the electric polarizability tensor of the molecule, $E_{0}$ is the amplitude of the oscillating electric field, and $v_{0}$ is the frequency of the incident laser light. The electric polarizability tensor can be interpreted as a deformability of the electron cloud by an external field, and depends on the dimensions of the molecular bonds.

If the molecule is vibrating at a specific frequency $v_{k}$ for the molecular vibrational mode $k$, then the displacement from the equilibrium position of nuclear, or normal vibrational coordinate $Q_{k}$ can be written as:

$$
Q_{k}=Q_{k 0} \cos \left(2 \pi v_{k} t\right)
$$


where $Q_{k 0}$ is the amplitude of the normal vibration. Expanding polarizability $\alpha$ in a Taylor series with $Q_{k}$ about the nuclear equilibrium position and for small vibrations, and substituting in the above equation gives:

$$
\begin{aligned}
\alpha=\alpha_{0} & +\frac{\partial \alpha}{\partial Q_{k}} Q_{k}+\ldots \\
& \approx \alpha_{0}+\frac{\partial \alpha}{\partial Q_{k}} Q_{k 0} \cos \left(2 \pi v_{k} t\right)
\end{aligned}
$$

where $\frac{\partial \alpha}{\partial \mathrm{Q}_{\mathrm{k}}}$ is the rate of change of $\alpha$ with respect to the $k^{\text {th }}$ normal mode about the equilibrium position and $\alpha_{0}$ is the polarizability of the molecule at its equilibrium position. Substituting this into equation 2.1, the induced dipole moment can be written as:

$$
\begin{array}{r}
\boldsymbol{P} \approx \alpha_{0} E_{0} \cos \left(2 \pi v_{0} t\right)+\frac{\partial \alpha}{\partial Q_{k}} Q_{k 0} E_{0} \cos \left(2 \pi v_{0} t\right) \cos \left(2 \pi v_{k} t\right) \\
=\alpha_{0} E_{0} \cos \left(2 \pi v_{0} t\right)+\frac{\partial \alpha}{\partial Q_{k}}\left(\frac{Q_{k 0} E_{0}}{2}\right)\left\{\cos \left[\left(2 \pi v_{0}-2 \pi v_{k}\right) t\right]+\cos \left[\left(2 \pi v_{0}+2 \pi v_{k}\right) t\right]\right\}
\end{array}
$$

This equation suggests that the induced dipole oscillates, and therefore radiates light, at three different frequencies, where the first term corresponds to Rayleigh scattering at the original incident frequency $v_{0}$, the second term, known as anti-Stokes scattering, corresponds to frequencies $\left(v_{0}+v_{k}\right)$ and relates the outgoing scattered photons that experience an increase in frequency by an amount $v_{k}$. The third term, known as Stokes Raman scattering, corresponds to frequencies $\left(v_{0}-v_{k}\right)$, and is associated with a decrease in frequency of the resulting scattered photon.

At room temperature most of the molecules will be in ground state, therefore it is expected that the majority of the Raman scattering will be dominated by Stokes Raman scattering. The intensity ratio of the Stokes and anti-Stokes scattered light depends on the 
population of the vibrational ground and first excited states, and can be calculated using Boltzmann's equation [18]:

$$
\frac{I_{\text {stokes }}}{I_{\text {anti-stokes }}}=\left(\frac{v_{0}-v_{k}}{v_{0}+v_{k}}\right)^{4} e^{\frac{h c v_{k}}{T k_{B}}}
$$

where $h$ is Planck's constant, $c$ is the speed of light, $k_{B}$ is Boltzmann's constant, and $T$ is the absolute temperature. Equation 2.5 highlights the proportionality of the Raman intensity to the fourth power of the frequency of the scattered Raman radiation. In general the Raman scattering intensity can be expressed as [18]:

$$
I=C I_{0} \alpha^{2}\left(v_{0} \pm v\right)^{4}
$$

where $C$ represent a series of constants, and $I_{0}$ is the intensity of the incident radiation. Since the incident laser frequency is adjustable and the frequency differences between vibrational states remain constant (a property of the molecule), the scattered intensity is essentially proportional to the fourth power of the incident laser frequency. Therefore, a high power, high frequency laser is desirable for increasing the intensity of Raman scattering.

\subsection{Raman Shift}

A Raman spectrum is a plot of the intensity of Raman scattered radiation as a function of its frequency difference from the incident radiation (usually in units of wavenumbers, $\left.\mathrm{cm}^{-1}\right)$. This difference or change called the Raman shift is given by:

$$
\text { Raman Shift }=\frac{1}{\lambda_{0}}-\frac{1}{\lambda}=\frac{v_{0}}{c}-\frac{v}{c}=v_{0}{ }^{\prime}-v^{\prime}\left(\mathrm{cm}^{-1}\right)
$$

where $\lambda_{0}$ is the wavelength, $v_{0}$ is the frequency, and $v_{0}{ }^{\prime}$ (wavenumber) corresponding to the incoming light and $\lambda, v$, and $v^{\prime}$ correspond to the Raman scattered light. Because it is a difference value, the Raman shift is independent of the frequency of 
the incoming light. The Raman spectrum is unique for each chemical composition and can provide quantitative and qualitative information of the sample.

\subsection{Raman Spectroscopy}

Raman spectroscopy is a form of vibrational spectroscopy that offers major advantages in comparison to the other analytical techniques. It is a non-invasive and non-destructive method that requires minimal sample preparation. Raman spectroscopy provides a chemical fingerprint of the investigated compounds and yields quantitative information about the sample through the interaction of the material's molecules with an incident laser beam. The Raman spectrum of water is weak and unnoticeable, allowing good spectra to be acquired from species in aqueous solution [19].

Raman spectroscopy also has several additional advantages. Unlike other vibrational techniques, its operational wavelength range is usually independent of the vibrational modes being studied. Since Raman spectroscopy measures the shift in frequency from that of the excitation laser, it can be performed using any operating range from UV to NIR. It thus permits access to vibrational mode information normally associated with wavelengths ranging from $2-200 \mu \mathrm{m}$. This makes Raman ideal for the study of materials that have vibrational frequencies in the far-infrared that are otherwise difficult to reach [19].

Despite the advantages of Raman spectroscopy detailed above, there are clear disadvantages to this technique. One drawback is the low penetration depth, which limits the technique to examination of material at the surface level [2]. Another obstacle that must be overcome in Raman spectroscopy is the very low Raman signal obtained due to 
the low probability of Raman scattering. Moreover, the weak signal necessitates higher laser powers (tens to hundreds of $\mathrm{mW}$ ) and long acquisition times, to obtain a Raman spectrum whose signal-to-noise ratio is high enough for analysis. This limits the use Raman spectroscopy on living systems that move or that can be damaged by the high laser intensity [19]. In spite of these drawbacks Raman spectra acquired from the cells and tissues are still useful for diagnostic purposes. However, real time imaging is not feasible due to the low intensity of Raman signals.

There are several mechanisms that can be used to enhance the Raman signal. The two most important approaches are resonance-enhanced Raman spectroscopy (RS) and surface-enhanced Raman spectroscopy (SERS). These developments have made important steps toward maximizing the diagnostic accuracy and speed, and often rely on cost-effective solutions that are likely to be adopted into the healthcare services [3]. Details of these methods are beyond the scope of this thesis.

To perform Raman spectroscopy, a light source (typically a laser) is shone onto the sample. The scattered light from the sample is collected through a series of optical elements that lead to a spectrometer, where Raman scattered light can be separated into its component wavelengths and captured on a CCD detector. A typical basic schematic of a Raman spectroscopy system is shown in figure 2.2.

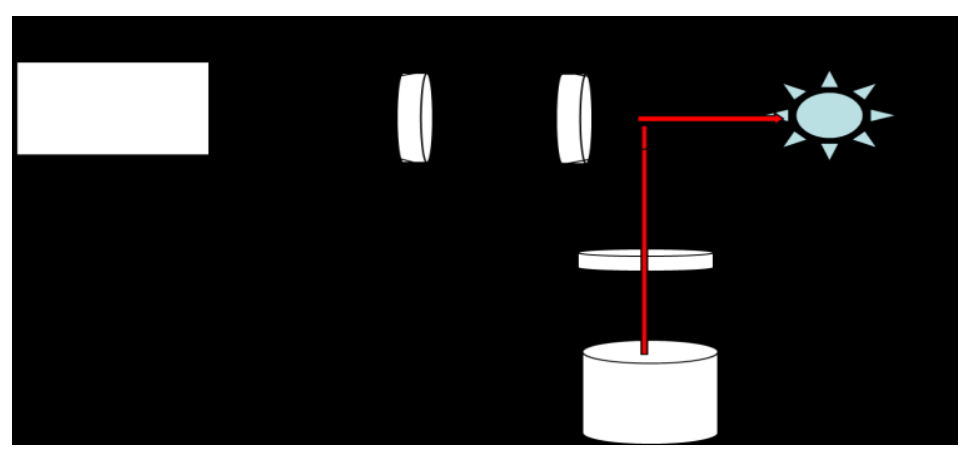

Figure 2.2: Basic schematic of the typical Raman spectroscopy system 


\subsection{Raman Micro-Spectroscopy}

Raman micro-spectroscopy is a technique that uses a specialized Raman spectrometer to measure spectra from microscopic samples. In general terms, such a spectrometer is integrated with an optical microscope. This is advantageous because it allows the sample to be resolved spatially, so that Raman spectra can be acquired from different parts of the sample with high precision [20]. A basic schematic of a Raman micro-spectrometer is shown in figure 2.3 .

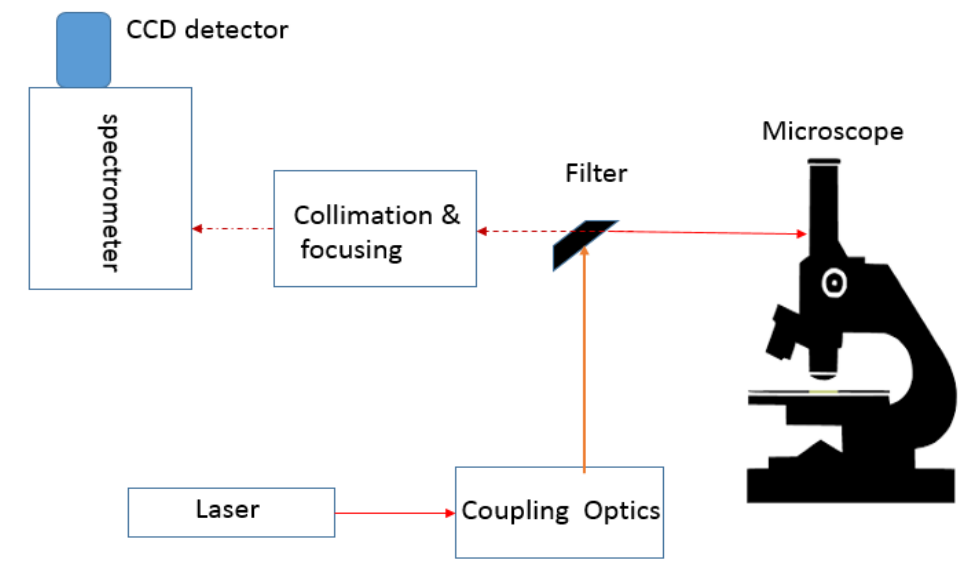

Figure 2.3: Basic schematic of the typical Raman micro-spectroscopy system

Typically the excitation source is directed into the microscope via a series of lenses and mirrors to guide, expand, and collimate the laser beam. The laser then passes through the objective and collides with the sample, which is positioned by an automated stage that offers precise spatial control. The reflected light from the sample is collected through the same objective, and falls on a Rayleigh filter that filters Raman scattered light, and excludes the Rayleigh scattered light. The filtered Raman scattered light enters the spectrograph and onto the CCD detector, producing the Raman spectrum. 
The specific Raman system used in this research will be discussed in detail in the following chapter, but the components of typical Raman micro-spectrometer are described in the following sections.

\subsubsection{Excitation Source}

Lasers have proven to be an excellent excitation source in Raman micro-spectroscopy, and they have become a standard component in a Raman system. Lasers provide a highly monochromatic source and an adjustable power option. The laser beam experiences minimal beam divergence and can be easily collimated and focused on a very small sample volume. There are three main parameters to consider when choosing a laser system: the type of laser source, the desired wavelength, and the desired spot size [20].

Typically continuous-wave (CW) diode lasers are used since pulsed lasers can provide extremely high instantaneous power, which increases the probability of damaging the sample and also results in other photochemical effects. On the other hand continuous-wave lasers provide frequency stability, with low instantaneous power, and a long operational lifetime [20].

The wavelength of the laser is critical to the type of experiment. Since Raman scattering intensity is proportional to $v^{4}$, where $v$ is the frequency of the laser, excitation at $400 \mathrm{~nm}$ leads to a 16 times higher Raman signal than say $800 \mathrm{~nm}$. However, many samples show strong fluorescence and photo-damage, when excited with a low wavelength source. Fluorescence emission can generate a large background signal and mask the weaker Raman spectrum. The least amount of fluorescence induced by laser light in the visible spectrum occurs for near infrared (NIR) wavelengths. The reduction in 
fluorescence at NIR wavelengths results in weaker Raman scattering, but also allows for longer acquisition times and the detection of weaker signals without damaging the sample [20].

The Raman signal is also proportional to the power and should be chosen well below the point where absorption would cause decomposition of the sample. The acceptable level of power depends on laser wavelength, sample properties such as thermal conductivity, and other imaging conditions like laser spot size. Practically speaking, it is better to have greater than $30 \mathrm{~mW}$ laser power at the sample for shorter visible wavelengths and greater than $100 \mathrm{~mW}$ for longer ones [20]. The total laser intensity (Power/area) is the central factor when acquiring high quality spectra, especially from biological samples, given that they are generally low-scattering materials that suffer from radiation damage $[20,21]$.

\subsubsection{Microscope Objective}

The choice of objective used in a Raman micro-spectroscopy system is crucial since it focuses the laser to a small spot on the sample and collects all the scattered light from the sample. Correct microscope objective maximizes the collection efficiency of the Raman scattered light and increase the spatial resolution of the system. In order to prevent potential fluorescence from the multiple optical lenses inside, the microscope objective must be of high optical quality [20].

A defining parameter of a microscope objective is the numerical aperture (NA), which describes the ability of optical system to collect light, and is given by [22]:

$$
N A=n \sin \theta
$$


where $n$ is the refractive index of the immersion medium and $\theta$ is the half angle of the maximum light cone collected by the objective as shown in figure $\mathbf{2 . 4}$.

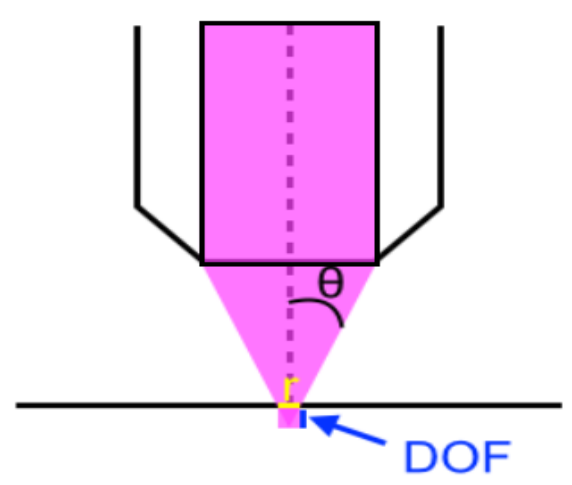

Figure 2.4: Illustration of the microscope objective with light cone. The angle $\theta$ is one half of the angle subtended by the cone. The dimensions of the laser spot are indicated by $\boldsymbol{r}$ and DOF referring to equations 2.9 and 2.10

The higher magnification and high NA objective (e.g. 100x, 0.8) provides higher axial resolution but shorter working distance, making it useful for thin samples. In contrast, low magnification and moderate NA objectives (e.g. 20x, 0.4) have lower axial resolution but longer working distances, and are better suited for bulky samples which do not require high spatial resolution. Conversely, high magnification is beneficial in studies that require high spatial resolution so that specific biological architecture can be examined.

The minimum beam waist radius of the focused laser spot depends on the NA of the objective and wavelength of incident laser and can be approximated by [21]:

$$
r=\frac{0.61 \lambda}{N A}
$$


The waist diameter $2 r$ is also called the spot size. The depth of penetration of the laser spot along the beam axis, or the depth of field (DOF) is also dependent on NA and defined by [21]:

$$
D O F=\frac{n \cdot \lambda}{N A^{2}}
$$

Both equations assume that the incoming laser is perfectly collimated and aligned with the back aperture of the objective. From the relationship between $r$, DOF and NA, maximum spatial resolution will be accomplished with the smallest focused spot, which can be achieved with a high NA objective and short wavelength.

Microscopic investigations of thinly cut fixed tissue sections and living cells adhered to substrates provide high-resolution images when employing objectives with a high numerical aperture (NA). Immersion fluid objectives provide a higher NA than air objectives, and are preferred because they give a better diffraction-limited spatial resolution, as was described earlier.

However, a significant amount of current biological research involves the investigation of cellular dynamics inside living cells or tissues. Important events relevant to these dynamics can occur deep within the specimen, away from the substrate. However, imaging with a conventional oil immersion objective is troublesome due to the significant florescence that will be observed when the laser goes through the substrate. Therefore, imaging must be done with a water-immersion objective that can be dipped directly into the cell medium so that the substrate can be avoided.

In Raman micro-spectroscopy, water immersion objectives are also preferred over oil immersion type since the oil gives rise to a series of Raman bands that can overlap the Raman bands of the sample. 


\subsubsection{Spectrometer and Light Dispersion}

Once Raman scattered light sample has been collected from the sample, the signal must be separated to different wavelengths to record the full spectrum. In most modern Raman micro-spectroscopy systems using visible or near infrared lasers, this is done using a dispersive spectrometer with a diffraction grating. The Czerny-Turner spectrometer is a common design and it consists of two concave mirrors and a reflective diffraction grating [16]. A schematic diagram of a basic Czerny-Turner spectrograph is shown in figure $\mathbf{2 . 5}$.

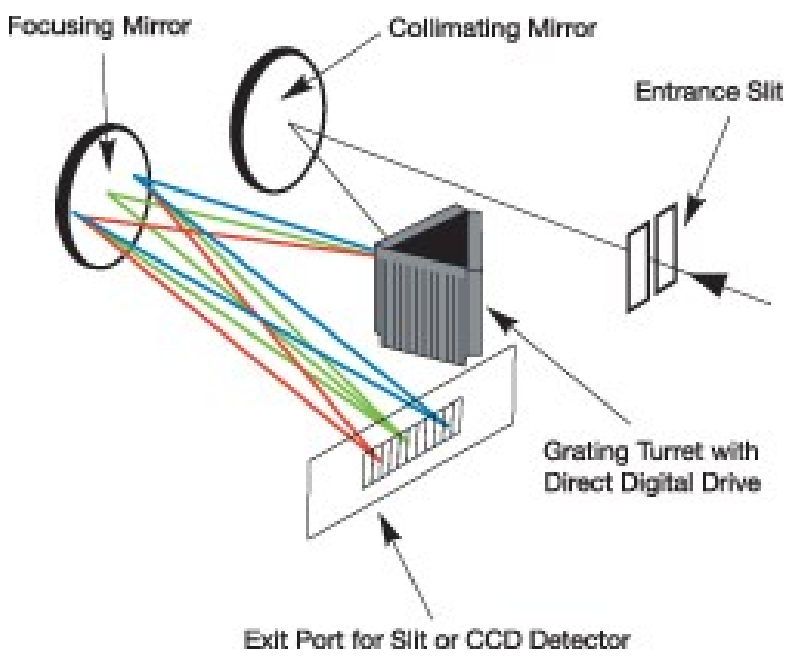

Figure 2.5: Schematic description of Czerny -Turner design for a spectrometer [www.azooptics.com/Article.aspx?ArticleID=309, by Andor Technology Ltd]

As illustrated in figure 2.5, the collected light enters the spectrometer through an adjustable slit, is collimated by a collection mirror and directed to a diffraction grating. The light is then reflected off of the grating at a specific angle that depends on the wavelength of the light according to [23]:

$$
\sin \alpha+\sin \beta=10^{-6} k n \lambda
$$

where $\alpha$ and $\beta$ are the angle of incidence and angle of diffraction respectively, $\lambda$ is the wavelength, $k$ is the diffraction order and $n$ is the groove density of the grating. 
Note the angle of diffraction is dependent on the wavelength of the light, and that allows the separation of different wavelengths of the scattered light.

The separated Raman scattered light coming from the grating is collected by the focusing mirror and focused onto the focal plane. The spatial distribution for the light emerging from the spectrometer is dependent on the linear dispersion of the grating. Linear dispersion defines the extent to which a spectral interval is spread out across the focal plane. The linear dispersion is expressed in terms $\mathrm{nm} / \mathrm{mm}$ (if using wavelength) or $\mathrm{cm}^{-1} / \mathrm{mm}$ (if using wavenumber), and is defined by the formula [23]:

$$
\frac{d \lambda}{d x}=\frac{10^{6} \cos \beta}{k n f}
$$

where $d x$ is the unit interval in $\mathrm{mm}$ and $f$ is the focal length of the focusing mirror, which is the arm length from the focusing mirror to exit slit. From the above equation, the linear dispersion is dependent on focal length and groove density of the grating. An instrument with better spectral resolution would have higher dispersion (a lower $\frac{d \lambda}{d x}$ ), which results in an increased spread-out of the spectral domain on the CCD. However there is a practical limitation to the size of the focal plane. Therefore, there is a limited spectral window that can be measured in a single acquisition. This can be increased by decreasing either the grating groove density or the focal length, but it is generally undesirable since the resolution of the spectrum would be compromised.

\subsubsection{Charge-Coupled Devices and Light Detection}

The detector is one of the key components of Raman micro-spectroscopy system and choosing the right detector strongly affects the performance of the instrument. To detect the weak intensity of the Raman scattered light, the detector must be extremely sensitive. 
A common detector used is the charge-coupled device (CCD). The CCD is placed at the focal plane of the spectrometer, and consists of a two dimensional array of light-sensitive silicon photodiodes, each connected to a capacitor.

In a photodiode, every detected photon creates an electron-hole pair which is separated by an internal electric field. The electrons generated from the pair manipulate the electrical charge on a capacitor that is converted into a digital value. An important characteristic of the detector is its quantum efficiency $(\mathrm{QE})$, which is the number of photo-electrons generated per photon that reaches the detector. As the light sensitive area of the detector is partially blocked by the electrical lines, the QE of a typical front illuminated (FI) detector is about $50 \%$ of its maximum. To increase the detection efficiency many detectors are available as back-illuminated (BI) devices, which are etched (back thinned) to about $15 \mu \mathrm{m}$ thickness. Thus, the electrons which are formed by the irradiation of the backside can migrate through the chip to the potential drains at the front of the detector where the electrical circuit printed. With this technique and proper anti-reflection coating a QE of more than $90 \%$ can be achieved. A comparison of QE curves of different type of CCD is shown in figure 2.6 [21,24]. 


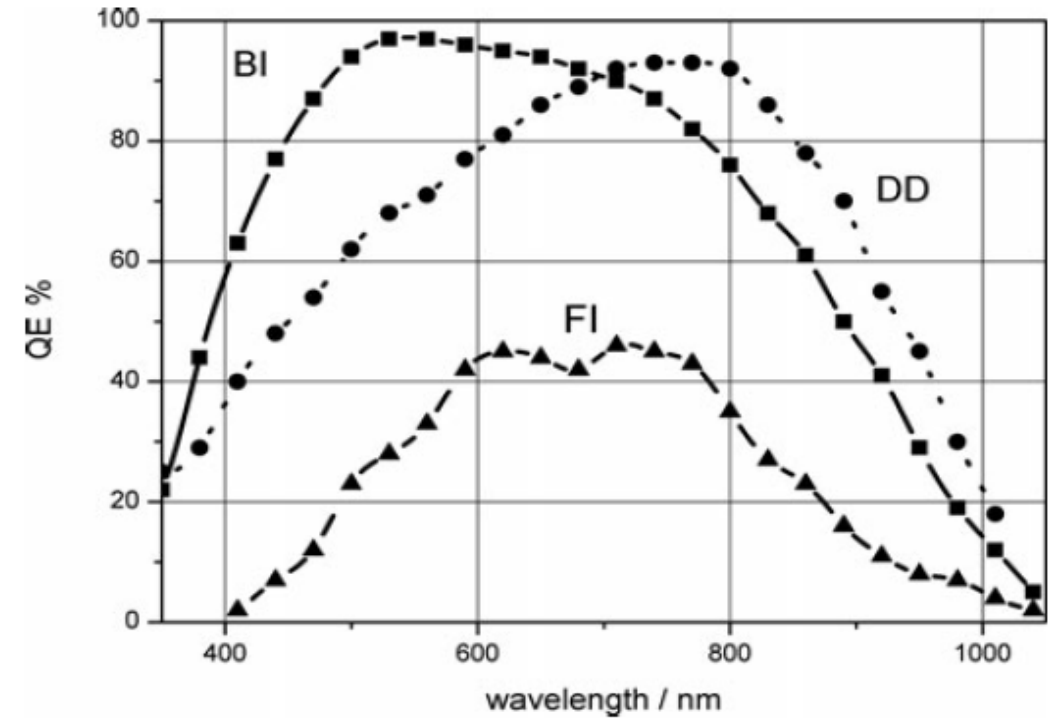

Figure 2.6: Quantum efficiency curves for back-illuminated (BI), back-illuminated deep-depletion (DD) and front-illuminated (FI) CCD detectors as a function of wavelength [21]

As can be seen from the graph, the quantum efficiency of silicon based CCD detectors is wavelength dependent and drops off rapidly in the near-infrared region (NIR). Another effect which is used to increase the sensitivity at relatively longer wavelengths is called deep depletion (DD). The term refers to the use of a doped silica chip, since doping increases the resistivity of the silicon. This allows the absorption of incoming photons to occur deeper in the device.

\subsection{Noise in Raman Spectroscopy}

The ability to extract analytical information from the Raman spectra is usually limited by the signal-to-noise ratio (SNR). Raman scattering is a very weak effect and the output spectrum is a combination of the signal from the sample, as well as the noise which does not contain relevant information. Noise can severely reduce or overwhelm the Raman signal and seriously influence the precision of the obtained results. 
The total noise level of a Raman peak can be represented as a standard deviation, $\sigma$, that contains contributions from various sources. This noise level can be represented by

$$
\sigma=\sqrt{\sigma_{s}^{2}+\sigma_{d}^{2}+\sigma_{r}^{2}+\sigma_{b}^{2}+\sigma_{f}^{2}}
$$

where $\sigma_{S}$ is the standard deviation of the signal due to uncertainty in counting statistics, $\sigma_{d}$ is the standard deviation of the detector dark current, $\sigma_{r}$ is the standard deviation of the readout noise, $\sigma_{b}$ is standard deviation of background fluctuations, and $\sigma_{f}$ is the standard deviation of the flicker noise. These standard deviations and their sources are explained in detail in the following paragraphs.

It is a basic rule in spectroscopy that all spectroscopic processes are subject to a certain uncertainty. This uncertainty on a given spectroscopic signal is fundamental and is given by [18]

$$
\sigma_{s}=\sqrt{S}
$$

where $S$ is the signal intensity. This type of noise is commonly referred to as shot noise. Shot noise is a fundamental property of the quantum nature of light and arises from statistical fluctuations in the number of photons emitted from the object. This noise source is unavoidable and always present in imaging systems. In terms of signal-to-noise ratio, the best a detector can do is to approach the shot-noise limit.

The primary noise sources in a CCD detector are thermal noise due to electron motion or dark current, and readout noise. Dark current is due to thermal fluctuations in the detector that lead to the generation of electron-hole pairs. Noise due to dark current can be problematic for long acquisition times, but can be reduced by cooling the detector to temperatures of $-60^{\circ} \mathrm{C}$ or below [21]. The readout noise arises from the process of 
reading out a pixel's accumulated charge. The readout noise is independent of acquisition time and can be reduced by some pre-processing technique such as averaging and smoothing [18].

"Background" is a fairly general term, but we will use it here to mean any detected photons arising from the laser and sample other than Raman photons from the sample at the frequency of interest. In particular, background includes luminescence from the cell, sample, or optics (e.g. fluorescence, thermal emission), and stray light. Stray light includes surrounding room light and any elastically scattered laser light that is not removed by filters or by the spectrometer itself. Background noise can be reduced by using a long exposure time and averaging across the acquired spectra, as was stated in the last section.

Finally, variations in laser intensity at both low and high frequencies will cause proportional variation in Raman scattering and in the measured Raman signal. This variation results in the flicker noise.

\subsection{Confocality}

Another important aspect of a Raman micro-spectroscopy system is its confocal configuration. As was stated in an earlier section, most samples supported by a substrate show fluorescence when excited with lasers, as a result of interactions between light and the electronic states in the sample and substrate. The efficiency of fluorescence can easily be orders of magnitude higher than that of Raman interactions. Therefore it is extremely crucial to reduce the fluorescence background, which can be done by using an excitation 
source at which the sample and substrate fluoresces minimally or by reducing the depth of the sample that is imaged.

Unfortunately it is not always possible to find a source at which there is no fluorescence due to a higher efficiency of the florescence process than Raman scattering. Confocal microscopy involves placing a small spatial aperture (such as pinhole) on the axis of collected light, somewhere prior to entering the spectrometer. The confocal aperture limits the collection of fluorescence to photons emitted from the focal plane by rejecting the signal coming from regions beyond the confocal plane, as can be seen in figure 2.7 .

As the confocal setup limits the detected light to the focal plane, it also allows the analysis of transparent samples in $3 \mathrm{D}$ and allows optical sectioning to be performed without cutting the sample. However, a smaller confocal aperture will increase the confocal resolution (reducing focal volume) and reduce the unwanted signals, but if it is too small it can reduce the amount of the Raman signal collected from the interest region. Most confocal Raman microscopes employ pinholes ranging from 10-200 $\mu \mathrm{m}$, depending on the sample thickness $[21,25]$.

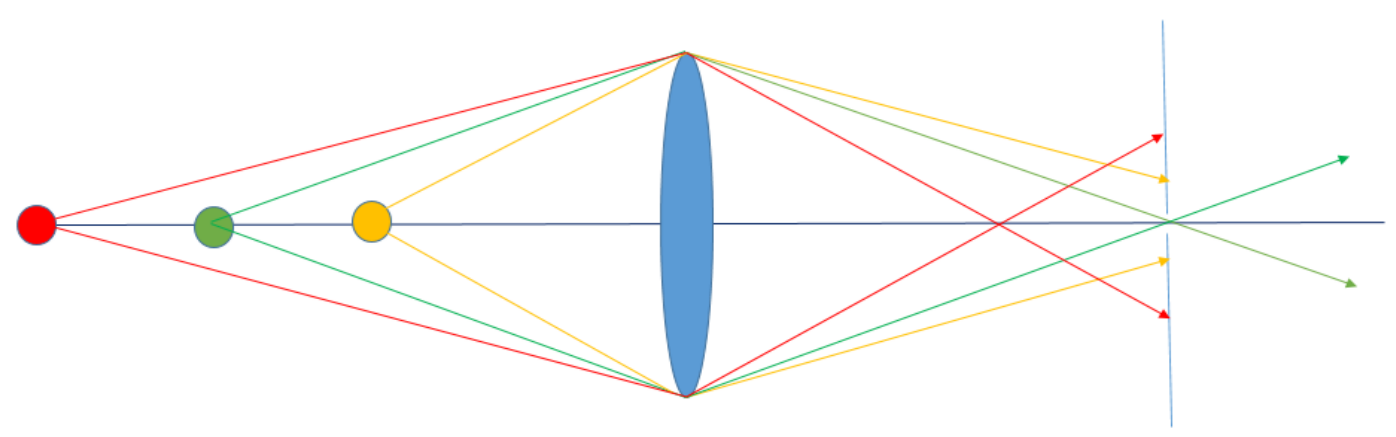

Figure 2.7: A diagram showing the pinhole function. The green dot is the light coming from focal plane and the red and yellow are the signal coming from out of focus planes [adapted from 26] 


\subsection{Issues in Biological Sample Measurements}

Raman spectroscopy has a number of properties which make it favorable for use in biomedical applications for investigations on tissue and single cells. It can be applied to samples in different physical states, and with a confocal microscope requires only small amounts of material, with minimum sample preparation.

Some considerations in relation to tissue and cell Raman microscopy must be addressed. In this section these considerations are explained in general, and details specific to the thesis project can be found in the next chapter.

The first parameter in biological application is the choice of the excitation source wavelength and power. The wavelength and power are considered in relation to the biological fluorescence and laser-induced damage to the cells. The biological fluorescence is minimized by using lasers with wavelengths in the upper visible or NIR region. This reduction in the sample fluorescence comes with an undesirable reduction in Raman scattering but allows for longer acquisition times, and weak Raman signals to be detected. There has been extensive study on the effect of the laser wavelength, power and exposure time on cell degradation $[27,28]$. These studies have shown that the cells exhibit no spectral changes for longer wavelengths with high power and longer acquisition, and the best laser wavelength range available for single cell Raman microscopy is $750-830$ $\mathrm{nm}$.

The second most important parameter is spectral range. There are two spectral ranges of interest for biological samples. The primary spectral window of interest is 450$1800 \mathrm{~cm}^{-1}$, and contains various Raman active normal modes from all cellular components: proteins, nucleic acids, carbohydrates, and lipids. The secondary spectral 
region of interest is $2400-3600 \mathrm{~cm}^{-1}$, which contains Raman shifts from lipids and proteins [5].

The other crucial consideration when taking Raman spectra of biological samples is the choice of substrate material. In order to culture the cells such that they attach properly, the substrate must be insoluble in water, growth media, buffer solution or any other chemical used. The materials used as substrate must be transparent to incoming laser light, and should not have Raman peaks in the spectral region of interest.

If the substrate is within the sample volume of the laser, it has a potential to contribute background fluorescence. Therefore, a substrate with low background fluorescence is selected. A variety of substrate materials have been studied for use in Raman spectroscopy, including quartz and calcium fluoride $\left(\mathrm{CaF}_{2}\right)$, as seen in figure $\mathbf{2 . 8}$. [29]. It was found that $\mathrm{CaF}_{2}$ had the lowest fluorescence background, but Quartz was ultimately used in this study because $\mathrm{CaF}_{2}$ is an expensive substrate.

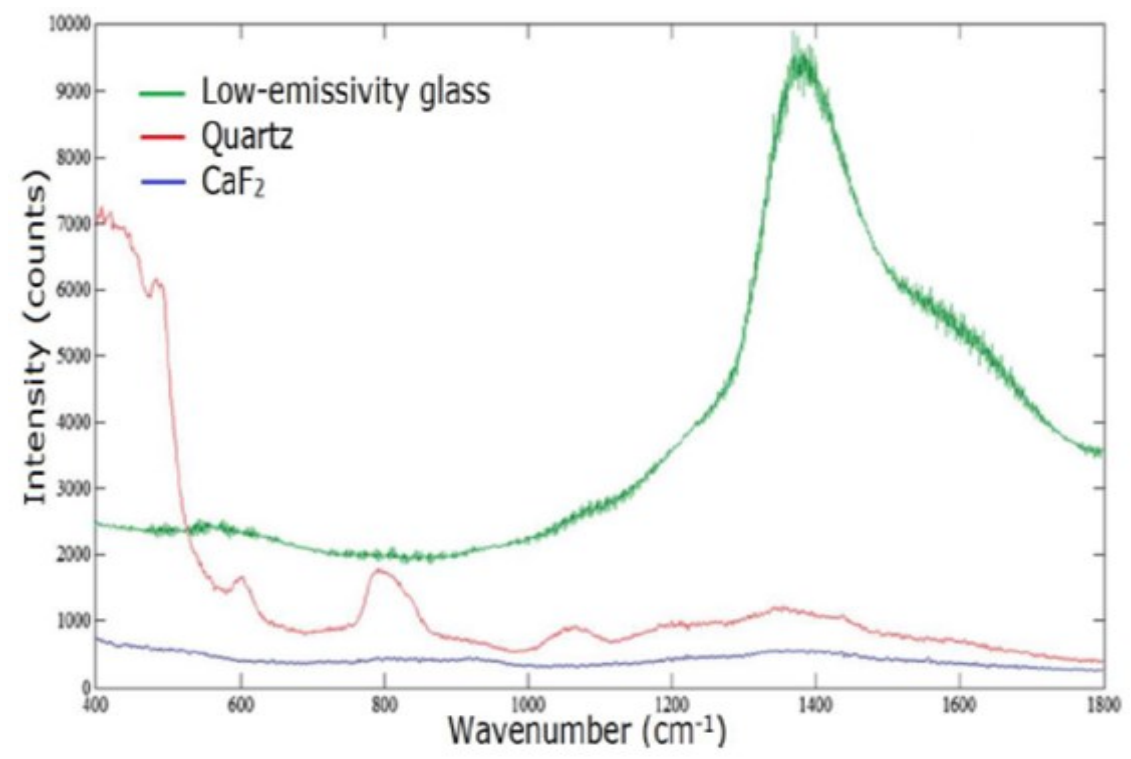

Figure 2.8: Comparison of spectra produced by $785 \mathrm{~nm}$ for different substrate materials [29] 


\subsection{Multivariate Statistical Analysis}

Multivariate statistical analysis refers to any statistical technique used to analyze data that arise from more than one variable. Due to the complexity of the cell Raman spectrum, which consists of many overlapping features, and the subtle differences between the spectra obtained from different samples (e.g. sensitive vs. resistant), extracting quantitative and qualitative information from the spectra is not straightforward. Very often, classical model assumptions are not fulfilled by spectral data, for instance there will be less observations than variables, or the variables are correlated. These problems have motivated the development of several methods for chemometrics. There are many different models, each with its own type of analysis, and two of these techniques are used to discriminate between sensitive vs resistant cancer cells. These techniques are described in the following sections.

\subsubsection{Principal Component Analysis}

The spectroscopic data can be displayed in the form of a matrix, where the rows represent the spectra and columns represent the wavenumbers. The reduction of dimensionality is necessary because we cannot effectively perform data classification if there are more variables than number of spectra. Our spectrometer camera is capable of acquiring spectra with 1024 data points (variables). In biological samples, there are a finite number of molecular compounds contributing to the Raman spectrum.

Principal Component Analysis (PCA) is a very effective technique for reducing the number of correlated variables, while preserving sample variability. This method takes a set of spectra which are potentially correlated and performs an orthogonal linear 
transformation of the data set into a set of "principal components", or new variables which are uncorrelated with each other. These principal components cumulatively contain/explain a large amount of variance within the data, and can be related back to the original variables via weighting factors. Now original variables with very low weightings factors in their principal components can be removed from the dataset since they do not generally hold much information about the variation within the data.

A rigorous derivation of the mathematical details on PCA can be found in Jollife [30], and only the main concepts will be explained in this thesis. Suppose Raman spectra have been obtained, each with intensities corresponding to different Raman shifts, or wavenumbers (1024 in this work). The spectra are the samples in this original data set, and the Raman shifts are the dimensions, or, using the language of linear algebra, the basis vectors of the original basis.

This spectral data can be arranged into a matrix, where the rows contain the samples (Raman spectra) and the columns contain the variables (wavenumbers). PCA seeks to re-express this data set in a new basis, whose basis vectors, called the "principal components" (PCs), are chosen in such a way as to reduce the number of dimensions (i.e. to reduce redundancy in the data) and to increase the signal-to-noise ratio (SNR) of the data [31,32]. There are three crucial assumptions in PCA: i) linearity, ii) large variances imply important structure in the data, and that iii) the PCs are orthogonal.

The first step in performing PCA is to subtract the mean of each dimension from each data point along that dimension. This produces data points along each dimension that have a mean of zero. The second step is to compute the covariance matrix of the data 
set. Considering the matrix defined above, we get the following expression for the covariance matrix:

$$
S_{X}=\frac{X * X^{T}}{n-1}
$$

where $\mathbf{X}$ are the mean-centred spectra in rows and $\mathrm{n}$ is the number of samples. This matrix contains the covariance between all pairs of dimensions. Large values in the diagonal terms, which are effectively variances, correspond to important structure (i.e. the signal) in the data, while large values in the off-diagonal terms correspond to dimensional redundancy in the data. Thus to minimize redundancy (measured by the magnitude of the covariance) and maximize the signal (measured by the variance), one must diagonalize the covariance matrix [31].

The third step is to compute the eigenvectors of $\mathrm{S}$ to obtain a diagonal matrix of the form:

$$
\Lambda=A S_{X} A^{T}
$$

where $\boldsymbol{A}$ is the orthogonal matrix containing the eigenvectors of $\boldsymbol{S}_{\boldsymbol{X}}$ each with length $p$, and $\boldsymbol{\Lambda}$ is the diagonal matrix containing the eigenvalues of $\boldsymbol{S}_{\boldsymbol{X}}$. The coefficients of the PCs are found as the eigenvectors of the covariance matrix and that is done by using singular value decomposition [31]. Since all the PCs form an orthogonal set, PCA can be considered as way of transforming the original data to, let's say a spectrum space, where the PCs are the new axes. In other words, the original axes show the intensity at specific wavenumbers, and the new axes describe the intensity of the contribution from specific PCs.

The PC score of each sample spectrum describes how much that PC is represented in the sample and can be determined by projecting the mean-centered spectrum onto PC 
axes. Each PC has one dimension, and the mid-point has value 0 . The PC loading vectors can be used to determine if particular wavenumber features are useful for discrimination. If the PC scores for a particular PC vector can be used to discriminate between classes, then the vector components with large magnitudes show that these components (features) yield a large contribution to the discrimination. The features can be related back to biochemical bonds to obtain physical information.

\subsubsection{Linear Discriminant Analysis}

While PCA is an unsupervised algorithm that determines the orthogonal axes that maximize the variance of the data, linear discriminant analysis (LDA) is a supervised algorithm that takes the class labels and tries to find the projection that maximize the ratio of between-class variance to within-class variance. Both algorithms are depicted in figure 2.9, where we see that projecting the samples onto the LDA line allows the two classes (red and blue) to be discriminated. LDA produces at most "C-1 (number of classes-1)" or "n (number of variables in the original data set)" solutions, whichever is lowest. In the context of spectroscopic data, LDA gives " $\mathrm{C}-1$ " projections, called Linear Discriminants (LDs) [31,33]. A rigorous mathematical treatment can be found in Reference 34 . 


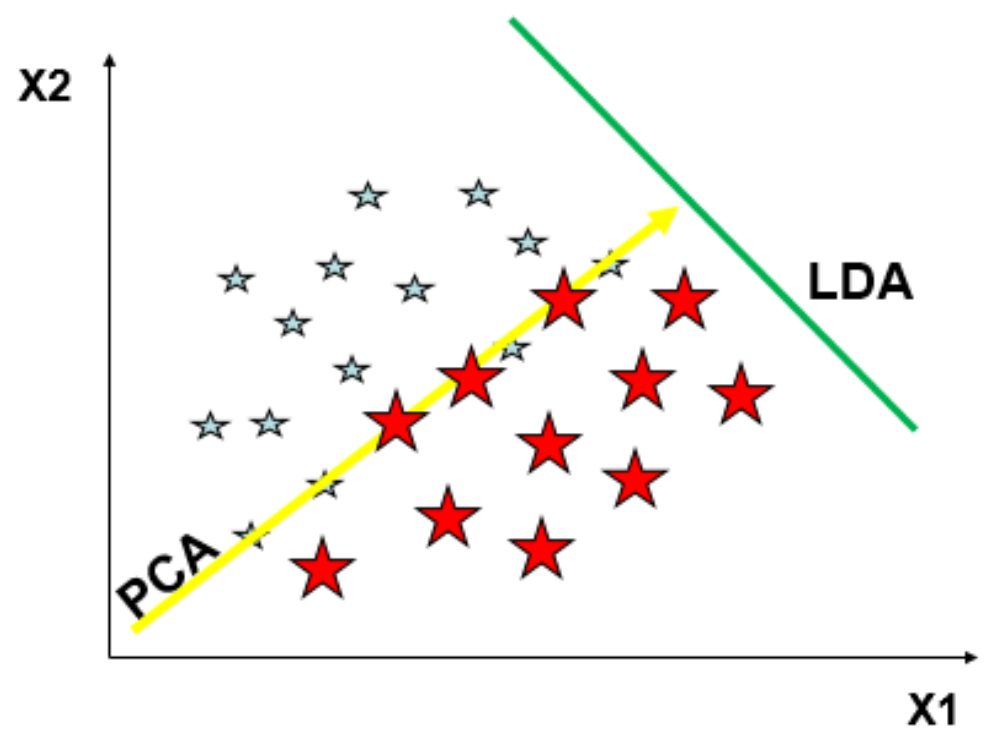

Figure 2.9: Schematic representation of axes found using PCA and LDA for two class data [adapted from 33]

LDA is combined with cross-validation methods to assess the predictive accuracy of the results. Assuming a dataset of two classes, the leave-one-out cross-validation (LOOCV) method involves removing a single sample from the dataset, and applying LDA to the rest of the data to build a classification model. The removed sample is then classified with the previously built-model. By iteratively applying this method on each sample in the dataset, the results can be summarized in a "confusion matrix" and a misclassification rate can be calculated as an accuracy estimate of the LDA model. This is called the "training phase". It is followed by a second phase, called the "prediction phase" or "testing phase", where the unseen data are predicted using the model parameters learned in the previous phase [33]. 


\section{Chapter: Instrument Development and Performance}

In the previous chapter we discussed the theoretical aspects of Raman microspectroscopy and related concepts used in this thesis. Now we move on to discuss the development of the Raman micro-spectroscopy system for cell imaging. This chapter presents the details of the setup components and processes used to build and characterize the instrument.

\subsection{System Overview}

In this work, Raman spectroscopy was implemented through a home-built confocal Raman micro-spectroscopy system as shown in figure 3.1.

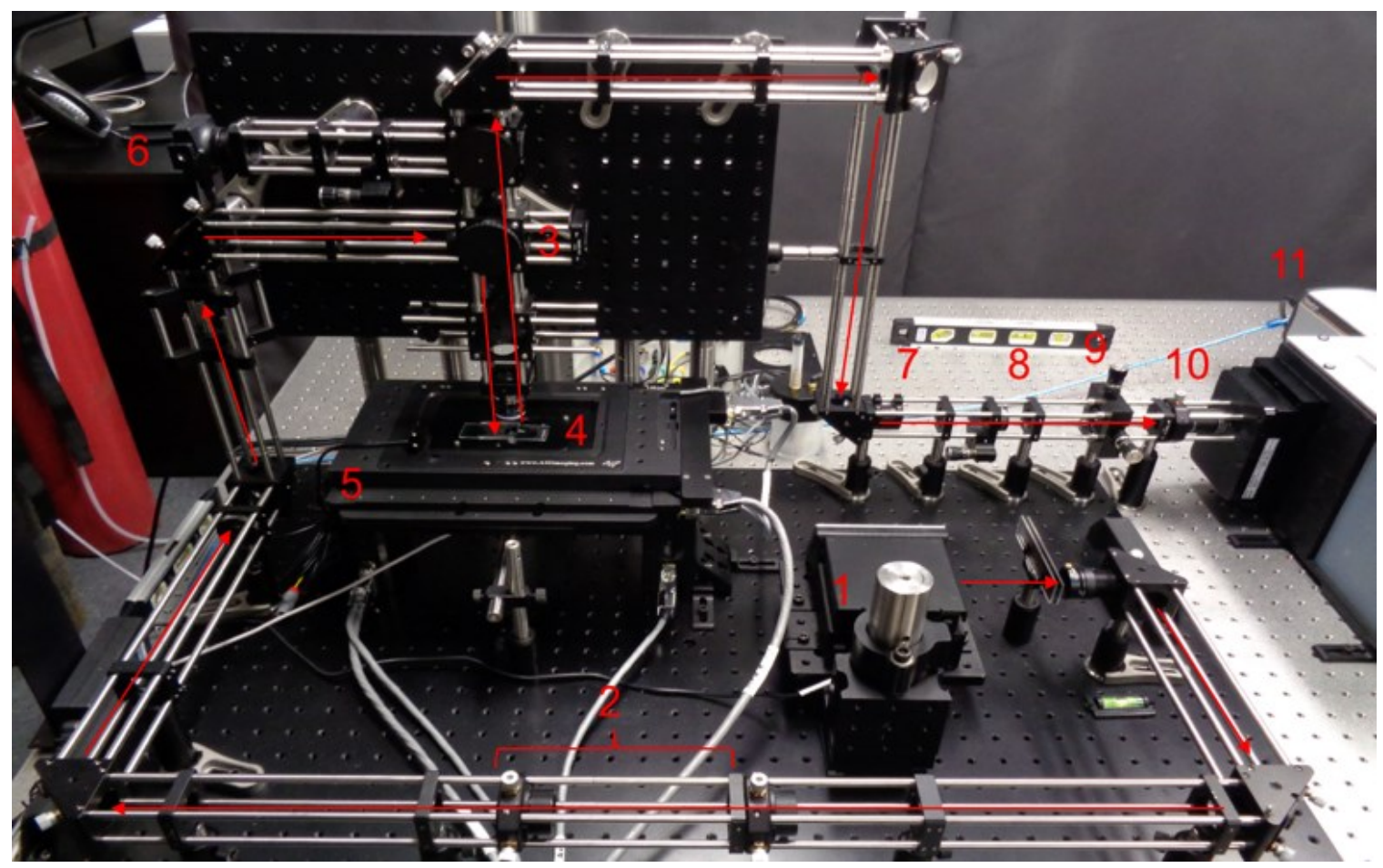

Figure 3.1: Custom confocal microscope coupled to a Raman spectrometer. (1) $785 \mathrm{~nm}$ laser (2)

Optics for laser beam collimation and expansion (3) Dichroic mirror (4) Microscope Objective (5) Automated x-y-z stage (6) CCD camera for bright-field imaging (7) Laser rejection filter (8) Pinhole lens (9) Pinhole (10) Focusing optics and (11) Spectrometer and CCD detector 
The major concerns when building a Raman system for the study of biological samples such as cells (fixed or live) are: collection efficiency, spatial resolution, cell damage and maintaining proper conditions for cells during the experiment. The selection of the components used for this setup are discussed in detail, while aiming to maximize collection of Raman scattered light and spatial resolution, and minimize damage to cells under proper conditions. The experimental setup has been continually modified and developed to optimize the instrumentation for the investigating of cells.

A continuous waveform (CW) $785 \mathrm{~nm}$ diode laser (Innovative Photonic Solutions with min power of $100 \mathrm{~mW}$ ) is used to excite Raman scattering (component 1 figure 3.1). The choice of laser wavelength is crucial for studying biological samples like cells and tissues. As was mentioned in the last chapter, two effects need to be considered when working with biological samples: cell photo-damage and excitation of fluorescence emission. The near-infrared wavelength of $785 \mathrm{~nm}$ is selected to avoid cell damage and minimize the florescence effect. This is a compromise, since longer wavelengths yield much weaker signals, so that longer integration times and high laser powers are required in imaging. The laser has a beam quality $\left(M^{2}<1.5\right)$ that allows the beam to be focused to a diffraction limited spot.

The laser beam with beam diameter of $2 \mathrm{~mm}$ is expanded to fill the back aperture of the microscope objective, with a diameter of $8 \mathrm{~mm}$. Beam collimation and expansion are achieved through two lenses (Thorlabs), with a 1 " diameter and focal lengths of $f_{1}=$ $40.0 \mathrm{~mm}, f_{2}=-100.0 \mathrm{~mm}$ (component 2 figure 3.1). The lenses are coated with an antireflection coating for $650-1050 \mathrm{~nm}$, and are mounted in the Galilean telescope configuration to provide a $4 \mathrm{x}$ beam expansion. This ensures that the objective pupil is 
filled by the laser beam in order to achieve the nominal NA of the objective. The laser beam is reflected downward by a dichroic beam splitter (component 3 figure 3.1) (Semrock Razor Edge) that reflects wavelengths shorter than or equal to $785 \mathrm{~nm}$ but transmits longer wavelengths with a high transmission efficiency (>90\%), as shown in figure 3.2. This downward reflected beam is guided into the objective (component 3 ,

\section{figure 3.1).}

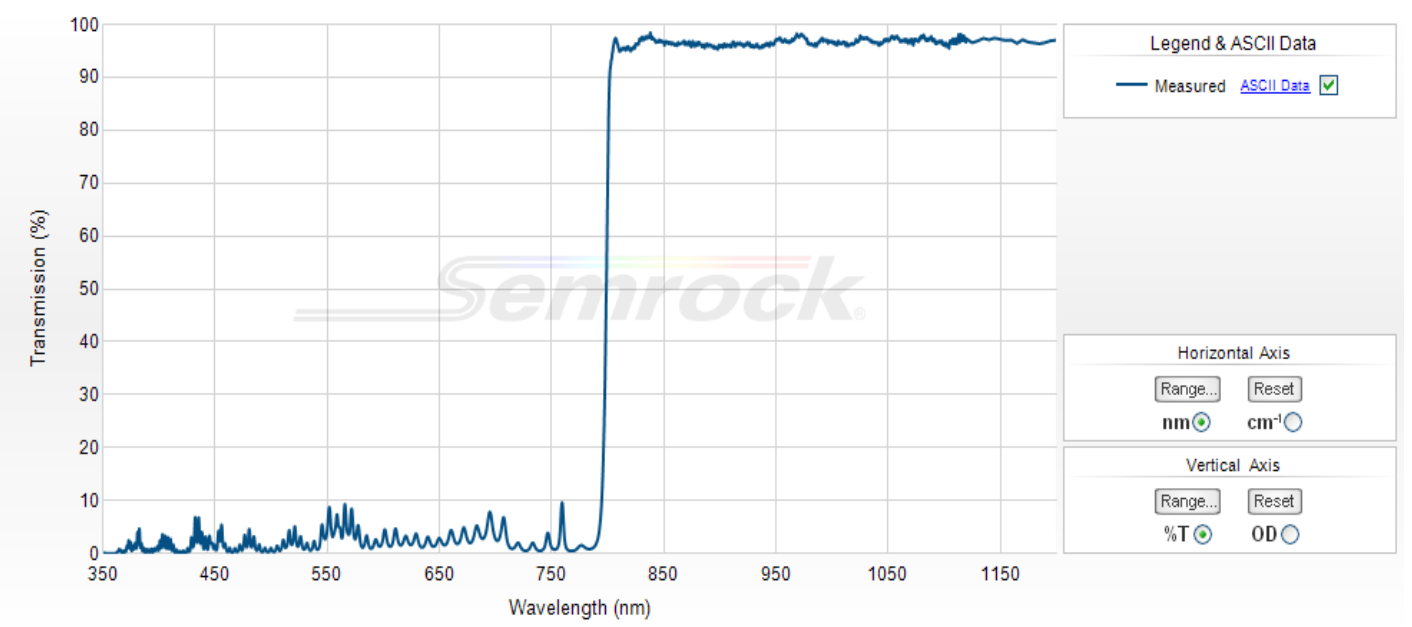

Figure 3.2: Transmission efficiency curve for dichroic beam splitter [www.semrock.com]

A water immersion microscope objective with an NA of 1.1 and working distance of $1.5 \mathrm{~mm}$ (LUMFLN 60x Olympus) is used to focus the laser beam onto the sample (component 4 figure 3.1). The laser beam delivers $35 \mathrm{~mW}$ of optical power to the sample plane with a spot size of $\sim 1 \mu \mathrm{m}$. The water immersion objective is selected over the oil immersion so as to minimize fluorescence from the quartz coverslip that arises due to the geometry of the system.

Back-scattered (epi-directed) light is collected by the same microscope objective, and the Raman-shifted light is transmitted through the $45^{\circ}$ dichroic filter (component 3 figure 3.1) described above. The collimated light is then relayed by a couple of mirrors to 
an ultra-steep Raman long-pass filter (component 7 figure 3.1) (IRIDIAN Spectral Technologies) to filter out any remaining laser excitation signal that has leaked through. The efficiency of this filter has been shown in figure 3.3.

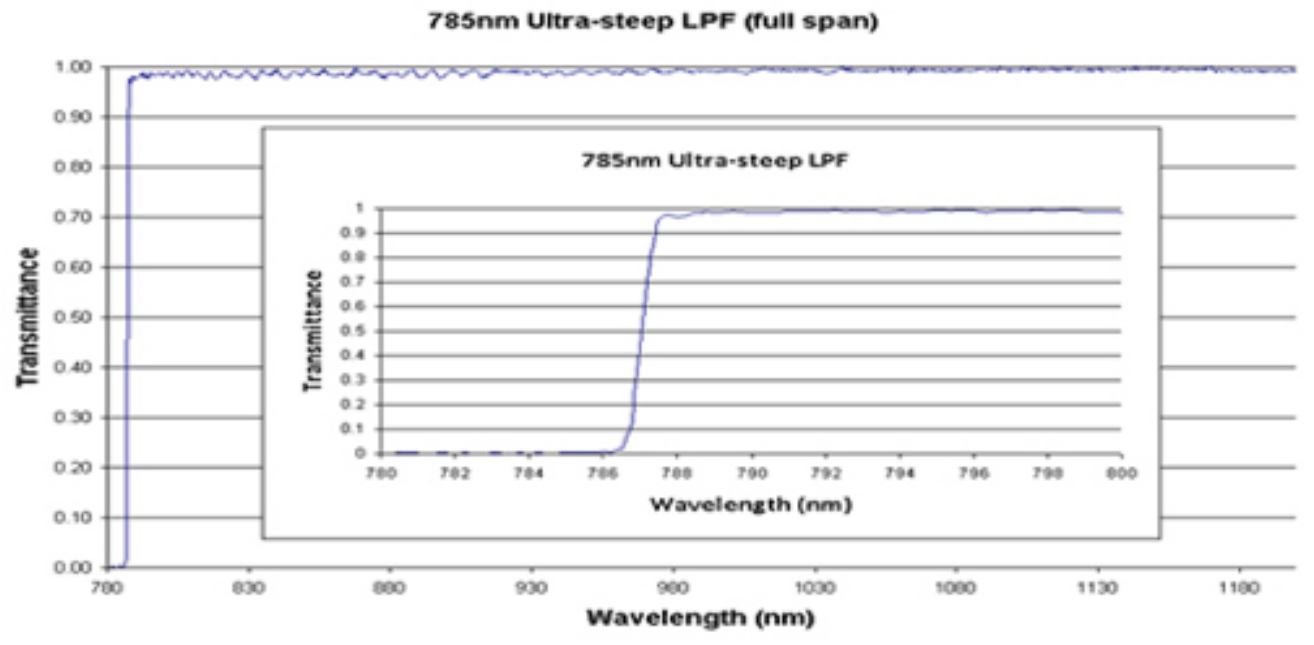

Figure 3.3: Transmission efficiency curve for long-pass filter [www.iridian-optical-filters.com]

As discussed in section 2.7, confocal collection is achieved through a pinhole located in front of the spectrometer to block out-of-focus light, so that light originating only from a small region of the sample is observed. Therefore a confocal microscope can achieve better axial resolution than a conventional microscope and is capable of imaging a thin section of the sample, referred to as optical sectioning. The pinhole diameter plays a decisive role in resolution and depth discrimination and depends on the desired spatial resolution and signal intensity. The smaller the pinhole is, the better the discrimination and the thinner the optical section. However a smaller pinhole yields weaker intensities of light. A compromise has to be made between the spatial resolution and signal intensity.

It has been shown that optical sectioning does not improve with pinhole size smaller than the radius of the first zero of the Airy disc [35]. A good approximation is to 
use a pinhole about the size of the Airy disk, but this makes the system difficult to align. The most effective solution is to enlarge the image significantly with the pinhole lens before casting it on the detection pinhole. The entrance of the pinhole is positioned in a plane conjugate to the microscope's sample plane. In the sample plane, the spot size of the focused beam is $\sim 1 \mu \mathrm{m}$, therefore a pinhole lens (component 9 figure 3.1) with focal length of $100 \mathrm{~mm}$ (Thorlabs) is placed where the beam is collimated, to magnify the laser spot on the pinhole. The choice of the pinhole size depends on the wavelength and the optical components used in the setup. The size is calculated using the following:

$$
D_{\text {pinhole }}=\frac{1.22 \lambda M_{t}}{N A}
$$

where $\lambda$ is the wavelength of the laser line at $785 \mathrm{~nm}, \mathrm{M}_{\mathrm{t}}$ is the total magnification of the system and NA is the numerical aperture of the objective. The total magnification depends on the focal length of the microscope objective, $f_{o b j}$, and the focal length of the pinhole lens, $f_{\text {pinhole lens }}$ and it is calculated using:

$$
M_{t}=\frac{f_{\text {pinhole lens }}}{f_{\text {obj }}}
$$

With $f_{o b j}=3.64 \mathrm{~mm}$ and $f_{\text {pinhole lens }}=100 \mathrm{~mm}$ used in our setup, the value of $\mathrm{M}_{\mathrm{t}}=28$. And similarly the numerical aperture of the objective depends on the proper filling of the pupil and the focal length of the objective through:

$$
N A=\frac{D_{\text {beam }}}{2 f_{o b j}}
$$

Using an objective with NA $=1.1$, pupil diameter of $D_{\text {beam }}=8 \mathrm{~mm}$ and $\mathrm{M}_{\mathrm{t}}=28$ in Equation 3.1, the optimal pinhole size is around $25 \mu \mathrm{m}$. As was mentioned above, in order to balance the signal intensity and the axial resolution, a pinhole of diameter 100 $\mu \mathrm{m}$ (Thorlabs) is selected (component 9 figure 3.1). 
The coupling of the microscope with the spectrometer involves intermediary optical elements that must match the numerical aperture of the spectrometer $\left(N A_{\text {spectrometer }}=0.125\right.$ or $\left.f / 4\right)$. A near IR achromatic doublet pair (Thorlabs) (component 10 figure 3.1) $f_{1}=40 \mathrm{~mm}, f_{2}=100 \mathrm{~mm}$ as is shown in figure 3.4, is used for the coupling to the spectrometer. The doublet pair was located $100 \mathrm{~mm}$ from the pinhole. The first lens will collimate light coming from the pinhole, and the second lens with a focal length of $40 \mathrm{~mm}$ will provide an $\mathrm{NA}=0.1$ (which is reasonably close to the required $\mathrm{NA}=0.125)$ to focus this light onto the spectrometer.
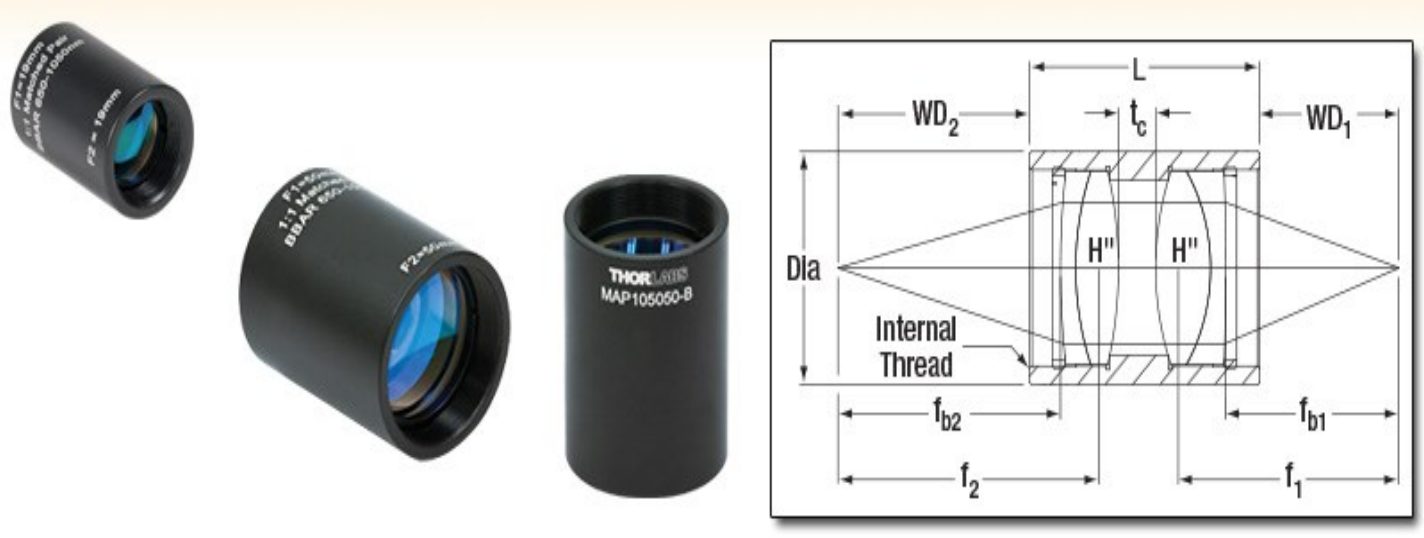

Figure 3.4: Optical coupling NIR achromatic doublet pair's schematic [www.thorlabs.com]

The spectrometer component 11 figure 3.1 used for our system is the Shamrock SR-303i with Czerny Turner configuration figure 3.5. It is a fully automated high throughput flat-field imaging triple-grating turret spectrograph. The focal length of the 
spectrometer is about $300 \mathrm{~mm}$. The choice of the grating is dictated by spectral resolution, spectral coverage and blaze efficiency.
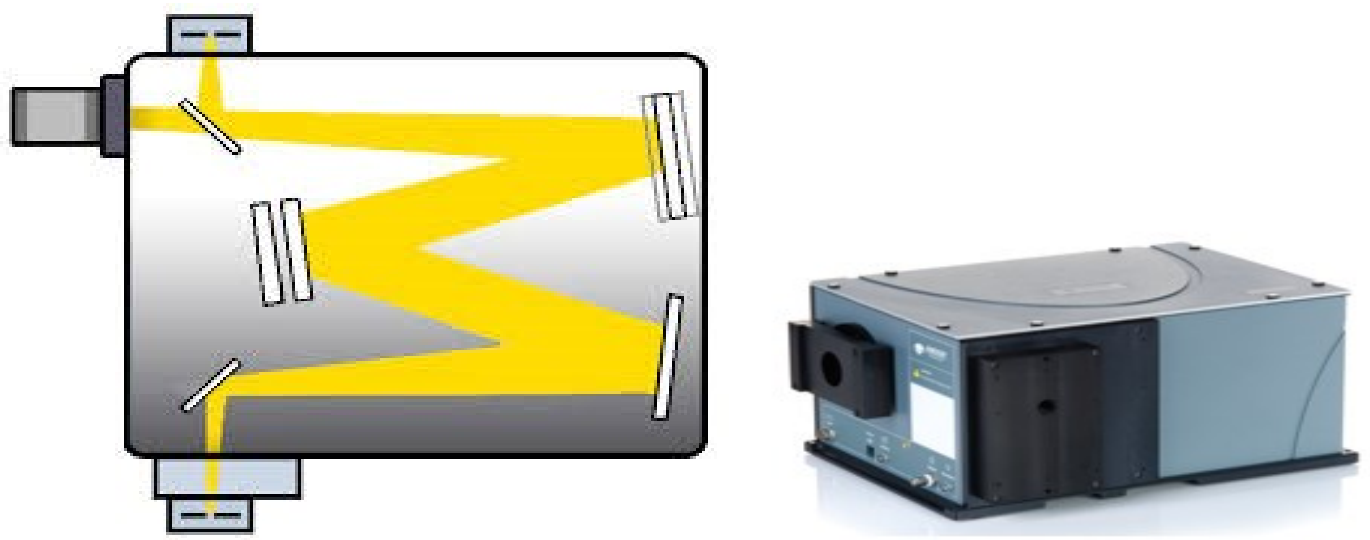

Figure 3.5: Shamrock SR-303i with Czerny Turner configuration [www.andor.com]

The higher the groove density of the grating (typically measured as number of grooves per millimeter), the higher the spectral resolution. In this study a grating with a groove density of 1000 lines $/ \mathrm{mm}$ with blaze wavelength of $900 \mathrm{~nm}$ is used, which is suitable for measurements of Raman spectral lines in the region 750-950 nm.

The CCD used in our system is Andor's thermally cooled back illuminated deep depletion CCD with anti-fringing, extended range dual AR coating (Newton DU920 BEX2-DD, Andor technology). This detector has an image area of $26.7 \times 6.7 \mathrm{~mm}$ with $100 \%$ fill factor, Active Pixels $1024 \times 256$ where each pixel has a dimension of 26 × 26 $\mu \mathrm{m}$. The detector has a maximum quantum efficiency over the desired range of wavelength in the near IR region, as shown in figure 3.6. Our system is thermally cooled to $-85^{\circ} \mathrm{C}$ prior to collecting spectra. 


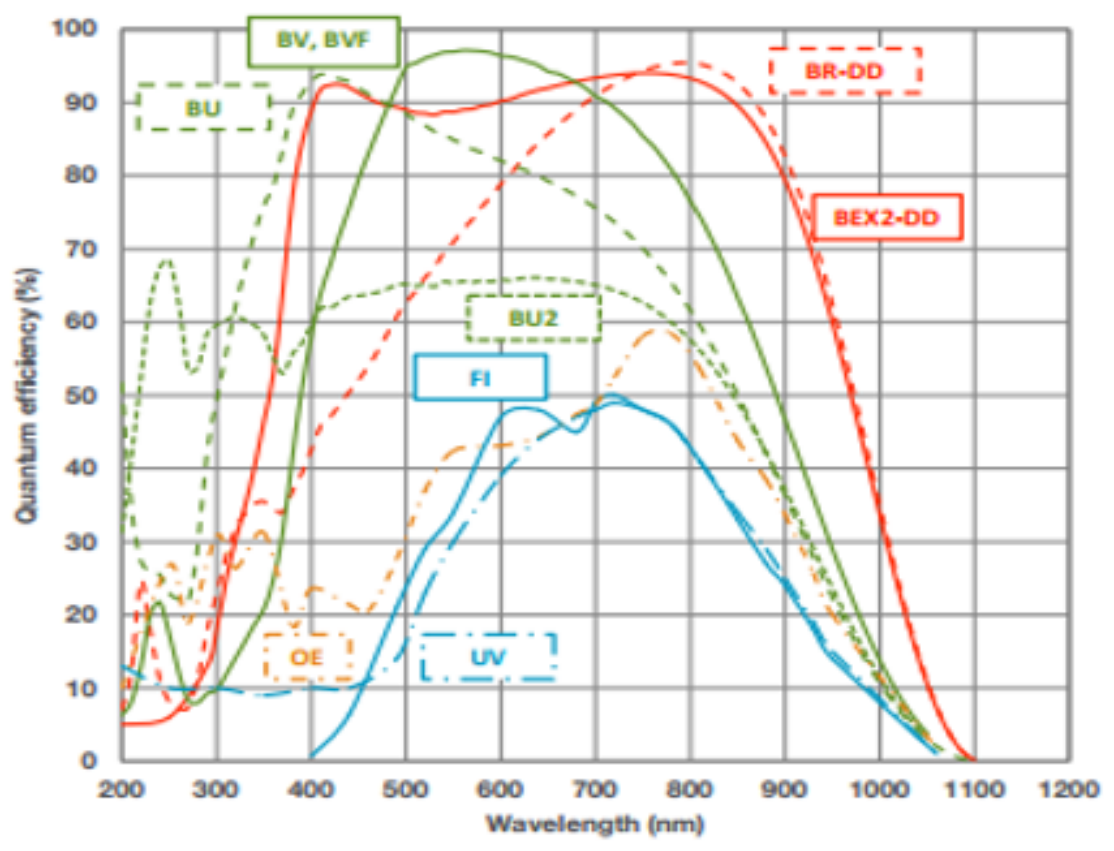

Figure 3.6: Quantum efficiency of CCD detector used in this system [www.andor.com]

A motorized electronically controlled automated stage (FTP 2000, ASI) was selected for precise positioning and highly repeatable focusing (component 5 figure 3.1). The unit is ideal for use with fixed stage microscopes, or any application where ultra-precise XYZ positioning is required. All axes derive their precise control through the use of closed-loop DC servomotors employing high-resolution rotary encoders for positioning feedback. By using closed-loop control of the stage position, there is no chance that the stage will become lost, as can occur with open-loop micro-stepped stages after a number of moves and direction changes. The microprocessor-controlled MS-2000 control unit provides for RS-232 and USB communication with a host computer. 


\subsection{Power Stability}

Fluctuations of the laser power could cause changes in the recorded photon numbers by the CCD camera and mislead interpretation of the concentration of the samples components. To verify the power stability, our laser was tested over several hours. Figure 3.7 shows the variation of the laser power recorded using the power meter (Thorlabs, PM100D) over six hours. The average value of the laser power within the six hour interval was $118.25 \mathrm{~mW}$. The standard deviation of $\pm 0.33 \mathrm{~mW}$ represents less than $0.3 \%$ of the average power value and indicates that the laser is suitable for Raman experiments over long period of acquisition time.

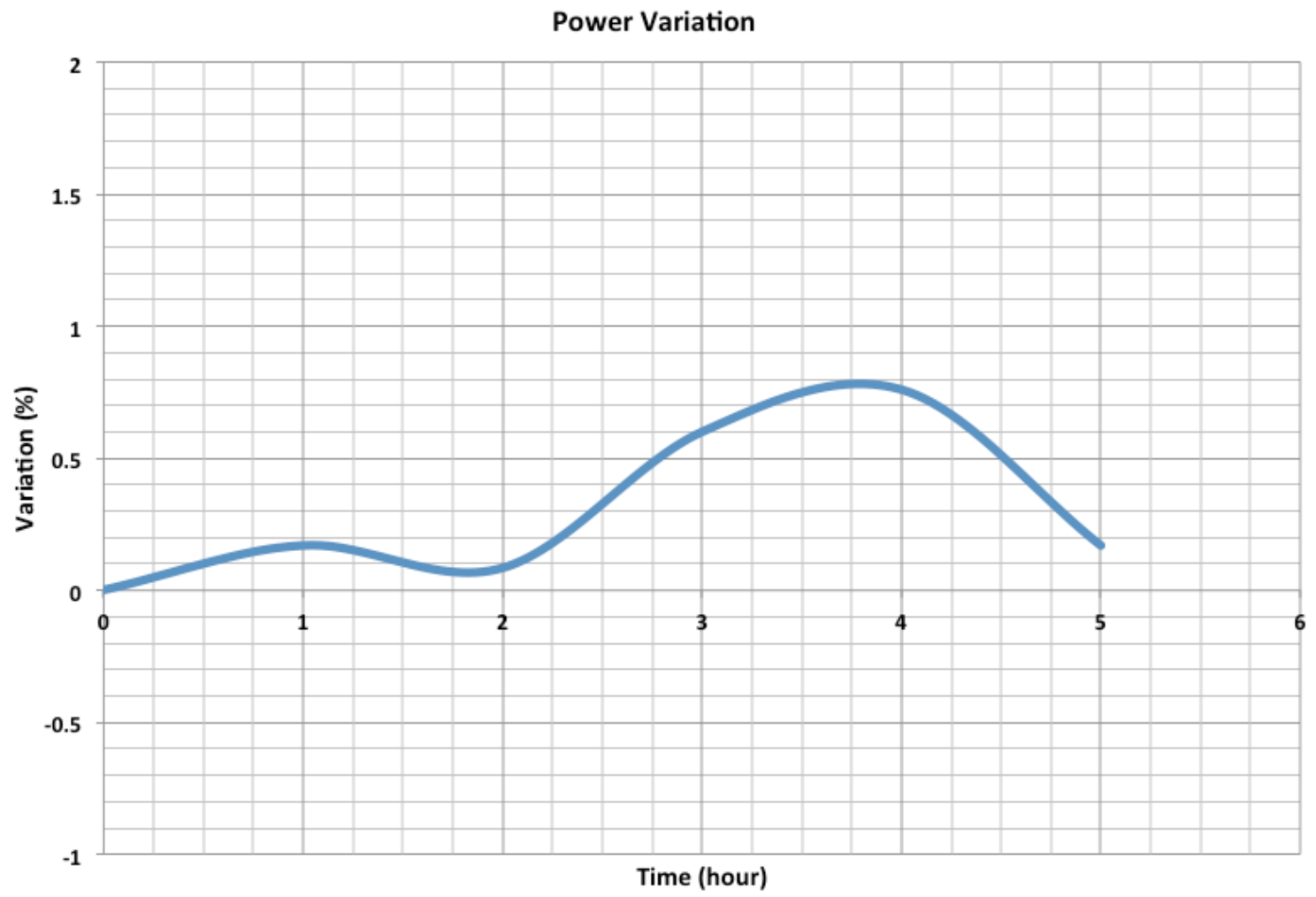

Figure 3.7: Power stability measurement for the laser 


\subsection{Spatial Resolution}

Spatial resolution describes the ability of an imaging system to resolve spatial detail in the object. The size of the cell is typically on the order of 10-100 microns and the cell organelles (e.g. Nucleolus, mitochondria) are on the order of 0.5-2 microns. Depending on the applications the selection of the spatial resolution of the laser spot is a crucial step in designing the confocal Raman micro-spectroscopy system. The study of sub-cellular contents, for example in nucleus, requires spatial resolution on the order of less than $1 \mu m^{3}$. On the other hand, studying the entire content of the cell requires spatial resolution on the order of the size of the cell. It is important to measure the spatial resolution of the Raman micro-spectrometer and its ability to distinguish features in both axial (z-plane) and lateral (xy-plane) dimensions. The resolution can be obtained experimentally in various ways [36].

\subsubsection{Lateral Resolution}

The resolution of a microscope objective lens is defined as the smallest distance between two points that can be distinguished. The achievable lateral resolution is primarily defined by the laser wavelength and the NA of the objective being used. The theoretical diffraction limited lateral resolution, according to the laws of optics, is defined by the following equation [25]:

$$
d \geq r_{\text {Airy }}=\frac{0.61 \lambda}{N A}
$$

where $\lambda$ is the wavelength of the laser, and NA is the numerical aperture of the microscope objective being used. The Rayleigh criterion states that if the distance between two equally bright spots, $\mathrm{d}$, is greater than or equal to the Airy radius, $r_{\text {Airy }}$, the 
points can be resolved. The calculated Airy radius for a $785 \mathrm{~nm}$ laser with a water immersion $\mathrm{NA}=1.1 / 60 \mathrm{x}$ objective would predict a spatial resolution of 435 nm. However, whilst this equation is applicable for standard light microscopy, the optical processes occurring during Raman microscopy are much more complex. For example, scattering of the laser/Raman photons and interaction with interfaces in the sample can reduce this resolution. Thus, typical Raman spatial resolution is often quoted as being in the order of $1 \mu \mathrm{m}[25]$.

\subsubsection{Axial Resolution}

Axial (z-axis) resolution is measured along the optical axis of the microscope, that is, perpendicular to the plane of focus. Axial resolution is more complex, and depends strongly on the confocal design of the Raman microscope being used. For a confocal design axial resolution of the order of $1-2 \mu \mathrm{m}$ is possible, allowing individual layers of a sample to be discretely analyzed. The achievable depth resolution will depend strongly on the laser wavelength, microscope objective, confocal hole aperture and sample structure. The theoretical resolving limit in the z-axis generated by a point-like object behind the pinhole is given by [37]:

$$
\mathrm{Z}=\sqrt{\left[\frac{0.88 \lambda_{e m}}{n-\sqrt{n^{2}-N A^{2}}}\right]^{2}+\left[\frac{n P H_{p r o j} \sqrt{2}}{N A}\right]^{2}}
$$

where $n$ is the refractive of index of immersion liquid, $\lambda_{\text {em }}$ is the emission wavelength, the projected $\mathrm{PH}, \mathrm{PH}_{\text {proj }}$, is the ratio between the physical pinhole size (100 $\mu \mathrm{m})$ to the total magnification $\left(M_{t}=28\right)$, and NA is the numerical aperture of the 
objective. The projected $\mathrm{PH}$ of our system is $3.6 \mu \mathrm{m}$ and the theoretical resolving limit in Z- plane of our system is approximately $\sim 6 \mu \mathrm{m}$.

For experimental axial resolution calculation, measurements were made on a polystyrene sheet (from petri-dish cover). The axial scan is performed by moving the sample throughout the laser's focus plane, starting from above the sample surface. The intensity of the Raman peak at $1001.4 \mathrm{~cm}^{-1}$ is measured with respect to z-position as shown in figure 3.8 .

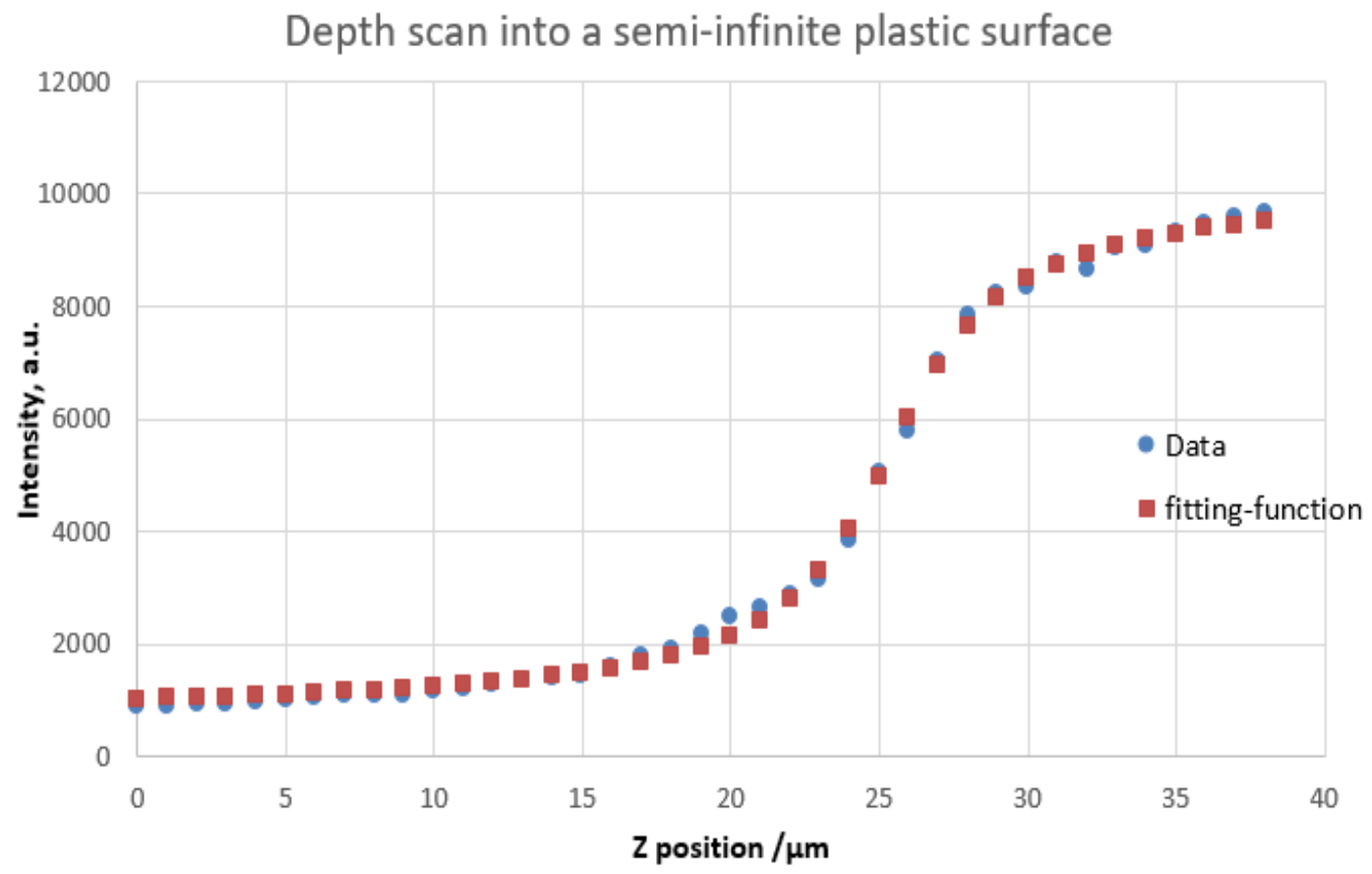

Figure 3.8: Experimental axial responses for a thick polystyrene sheet sample

In order to determine the axial resolution of the system we take the derivative of the intensity profile [62,63]. Figure $\mathbf{3 . 9}$ shows the derivatives of the step response curves. The axial resolution of the confocal setup is defined as the width of the response curve at $50 \%$ of the maximum signal, known as the full-width at half-max (FWHM). The 
experimental axial resolution of our system is found to be approximately $\sim 5.8 \mu \mathrm{m}$, which is very close to the calculated theoretical value.

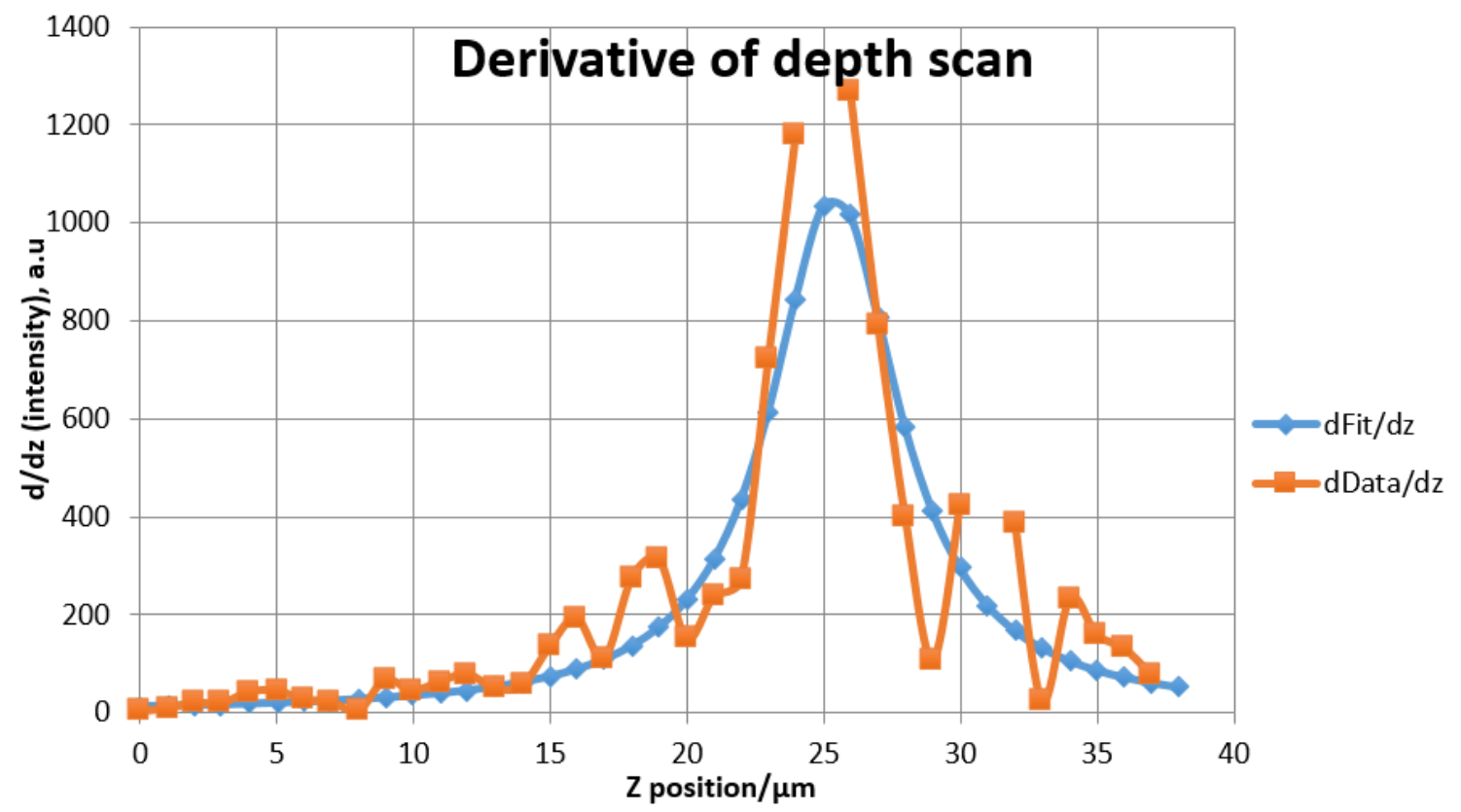

Figure 3.9: The derivative of the detected intensity function show in figure 3.8 for thick polystyrene sheet sample

\subsection{Spectral Resolution}

One of the most important characteristics of a spectrometer is its spectral resolution. The spectral resolution of a system is generally defined as the separation between the two closest peaks that the instrument can resolve.

In the most fundamental sense, both bandpass (BP) and resolution are used as a measure of an instrument's ability to separate adjacent spectral lines. Spectral bandpass specifies how much spectral bandwidth, FWHM, is being seen for a given wavelength position. Since this limits the ability of the spectrometer to separate peaks, it is common to refer to the bandpass as spectral resolution. The bandpass can be calculated by the following [64]: 


$$
B P=W \frac{d \lambda}{d x}
$$

where $W$ is the slit width in $\mu \mathrm{m}$ and $\frac{d \lambda}{d x}$ is the linear dispersion, which represents the number of wavelength intervals (e.g. $\mathrm{nm} / \mathrm{mm}$ ) contained in each interval of distance (CCD Pixel) along the focal plane. The dispersion is simply dependent on the spectral range (SR), size, and number of detector elements in the focal plane. It is given by [64]:

$$
\frac{d \lambda}{d x}=\frac{S R}{n \times \text { pixel size }}
$$

where $n$ is the number of pixels in the detector. For our system, $n$ is 1024 and the pixel size is $26 \mu \mathrm{m}$. SR depends on the grating resolving power, and varies with the grating used.

From the above equations, we conclude that there are five main factors that determine the spectra resolution of a spectrometer: laser wavelength, the spectrometer focal length, entrance (slit width), the diffraction grating (lines/mm), and the detector. Spectral resolution decreases as the excitation wavelength is changed from infra-red to ultra-violet wavelengths. The longer the focal length (the distance between the dispersing grating and detector) of the spectrometer, the higher the spectral resolution. The slit determines the minimum image size that the optical bench can form in the detector plane. The diffraction grating determines the total wavelength range of the spectrometer. The detector determines the maximum number and size of discreet points in which the spectrum can be digitized. Finally, the smaller the pixel, the higher the achievable spectral resolution.

The FWHM of the instrumental profile was measured experimentally by measuring the FWHM of the Raman band at $1001.4 \mathrm{~cm}^{-1}$ for a 5 micron polystyrene bead, as shown in figure 3.10. The overall spectral resolution can be calculated as the 
FWHM value of the Gaussian fit over the peak. The experimental spectral resolution of our system was determined to be $\sim 5.2 \mathrm{~cm}^{-1}$.

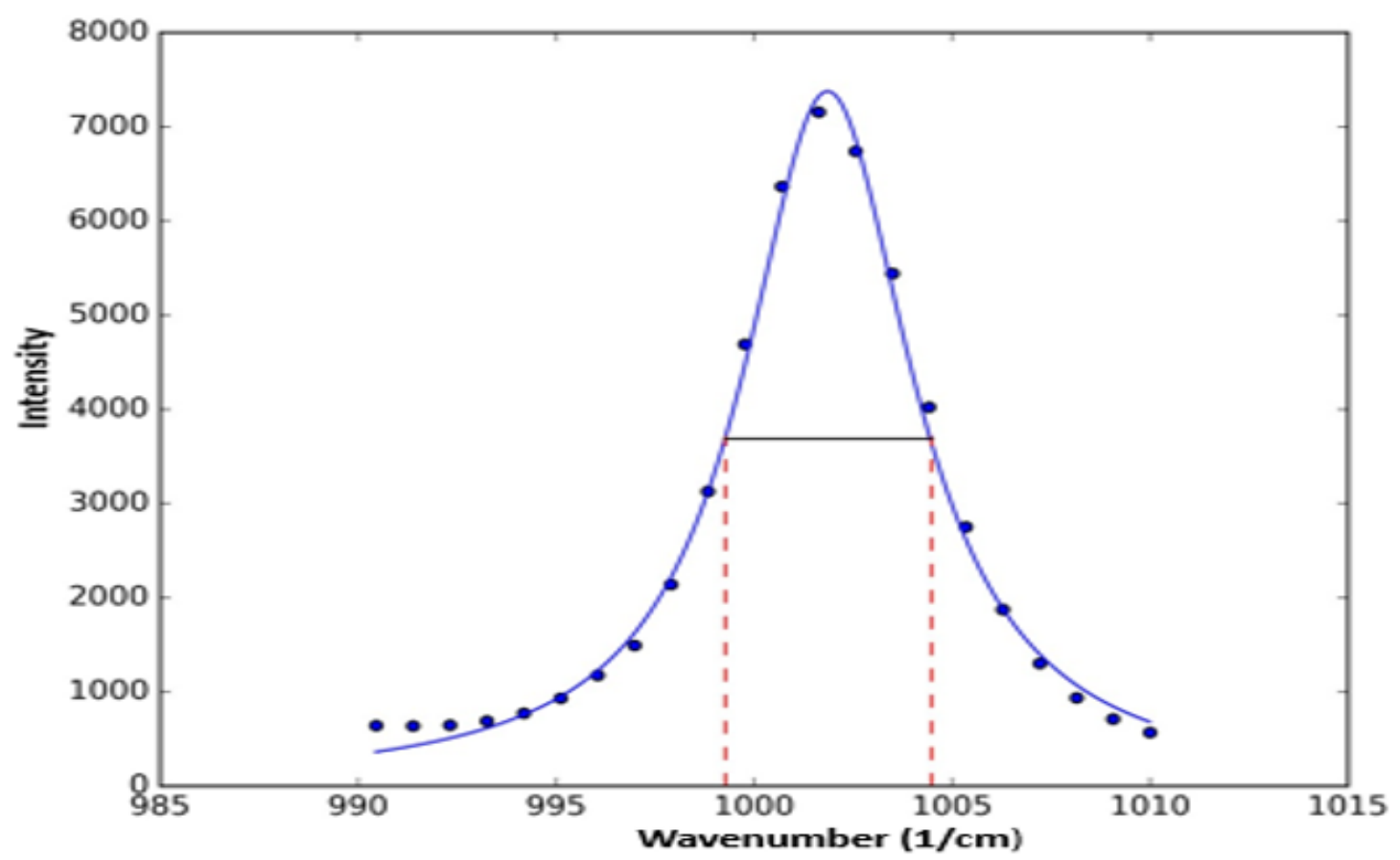

Figure 3.10: Spectral peak at $1001.4 \mathrm{~cm}^{-1}$ of polystyrene bead and fit to calculate FWHM to measure the spectral resolution of the system 


\section{Chapter: Materials and Methods}

This chapter details the materials and experimental methods used throughout the work presented in this thesis. The first part of this chapter presents the type of cell lines used in this work, and the general protocol for cell culture and preparation for Raman spectroscopy. This part of the project was carried out by Nhung (Rose) Vuong from Dr. Vanderhyden's lab. The second part describes the Raman spectroscopy protocol used for data acquisition and in the final part spectral preprocessing and data analysis methods are outlined.

\subsection{Cell Preparation}

Details of cell lines, the preparation and fixation protocol of the cell samples are discussed in the following subsections. These samples were prepared in Dr. Vanderhyden's lab at The Ottawa Hospital Cancer Center and transported to our lab at Carleton University.

\subsubsection{Cell Lines}

Cancer cell lines used in this study were derived from the A2780 human ovarian carcinoma cell line. The parental A2780s line was established from an untreated ovarian carcinoma patient, and the variant A2780cp line has been established by intermittent $(100 \mu M)$ exposure to cisplatin. The A2780s/A2780cp cell lines have been previously shown to be sensitive/resistant to radiation and their radiobiological behavior has been extensively studied [38]. The two cell lines thus represent a good model of tumor tissues of similar origin but with different intrinsic chemo and radio-sensitivities. 


\subsubsection{Cell Fixation}

In this work in order to collect spectra from the cells, it was necessary to chemically fix the cells on a quartz substrate. The fixation procedure kills the cells, but essentially preserves their internal structure. Selecting the ideal sample fixation method for sample analysis is critical for obtaining both sensitive and reproducible results.

Several methods of cell fixation exist and have been studied $[39,40]$. These studies compared the effects of three commonly used fixatives: neutral-buffered formalin, Carnoy's fixatives and a methanol-glacial acetic acid mixture. The level of fixation effect was found to be cell line dependent, and in general all the fixation methods were observed to affect the vibrational modes of lipid, protein, nucleic acid and carbohydrate moieties. The results suggest that the formalin preserves the spectral content that resembles the live cells and therefore best preserves cellular integrity. Hence this method was used for cell fixation in this work.

\subsubsection{Cell Culture and Preparation}

The cells were prepared by Dr. Vanderhyden's lab as follows. The A2780s and A2780cp cells were purchased from the European Collection of Authenticated Cell Cultures and cultured in Dulbecco's Modified Eagle Medium with $10 \%$ fetal bovine serum at $37^{\circ} \mathrm{C}$ and $5 \% \mathrm{CO}_{2}$. Approximately $5 \times 10^{5}$ cells of each subtype were seeded onto two separate quartz coverslips and were allowed to attach overnight in the incubator. The media were aspirated and the coverslips were washed with phosphate buffered solution (PBS) three times and then fixed with $4 \%$ formalin for 10 minutes at room temperature. The 
coverslips with the chemically fixed cells were immersed in PBS for imaging and storage. Raman spectra from both cell types were collected on the same day.

\subsection{Spectral Acquisition}

Raman spectra were acquired from 30 to 40 randomly selected cells from both types of sensitive and resistive cells in a day. The back-scattered Raman signal was integrated for 30 seconds over the spectral range of 700 to $1680 \mathrm{~cm}^{-1}$ for each selected cell and averaged over 10 accumulations to improve signal-to-noise ratio. A total of 223 spectra (110 A2780S cells and 113 A2780CP cells) were collected over the 4 different days. A variety of cell sizes, shapes and locations were selected to minimize potential biases in cell selection. The bright-field image on the CCD camera (Thorlabs) was used to locate the cells and then the laser was focused on the surface of the quartz cover slip, as can be seen in figure 4.1. To acquire data from the cell, the stage was moved down by a few microns (e.g. $4 \mu \mathrm{m}$ ) to bring the cell in the focal plane of the laser. For background subtraction from the cell spectra, Raman spectra were collected from nearby regions that did not include cells. 


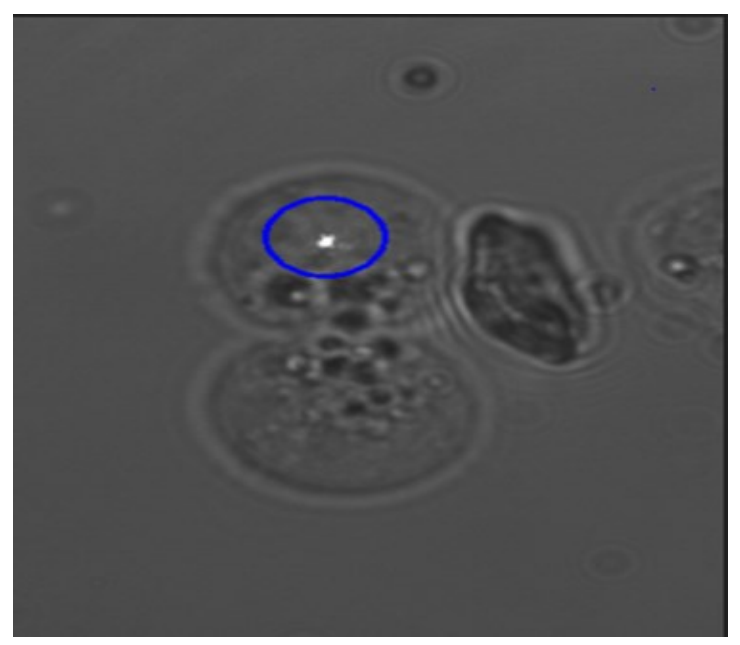

Figure 4.1: Bright field image of fixed ovarian cancer cell with the bright white spot being the laser

As a check at the beginning and the end of data collection for each day, a silicon reference spectrum centered at $520.0 \mathrm{~cm}^{-1}$ is collected, as shown in figure 4.2. Any obvious problems in laser or system alignment that would affect laser intensity at the sample would be detected in this reference silicon peak intensity and location. The average initial silicon peak intensity over all experiments was 33000 counts $\pm 4 \%$ (over one second integration) and the average silicon peak location was $520 \pm 0.4 \mathrm{~cm}^{-1}$, suggesting that the laser power at the sample was relatively consistent throughout all experiments. Prior to data acquisition the cell is exposed to 10 seconds of photobleaching where the cell is irradiated with the laser in order to quench background fluorescence before the spectrum is acquired. 


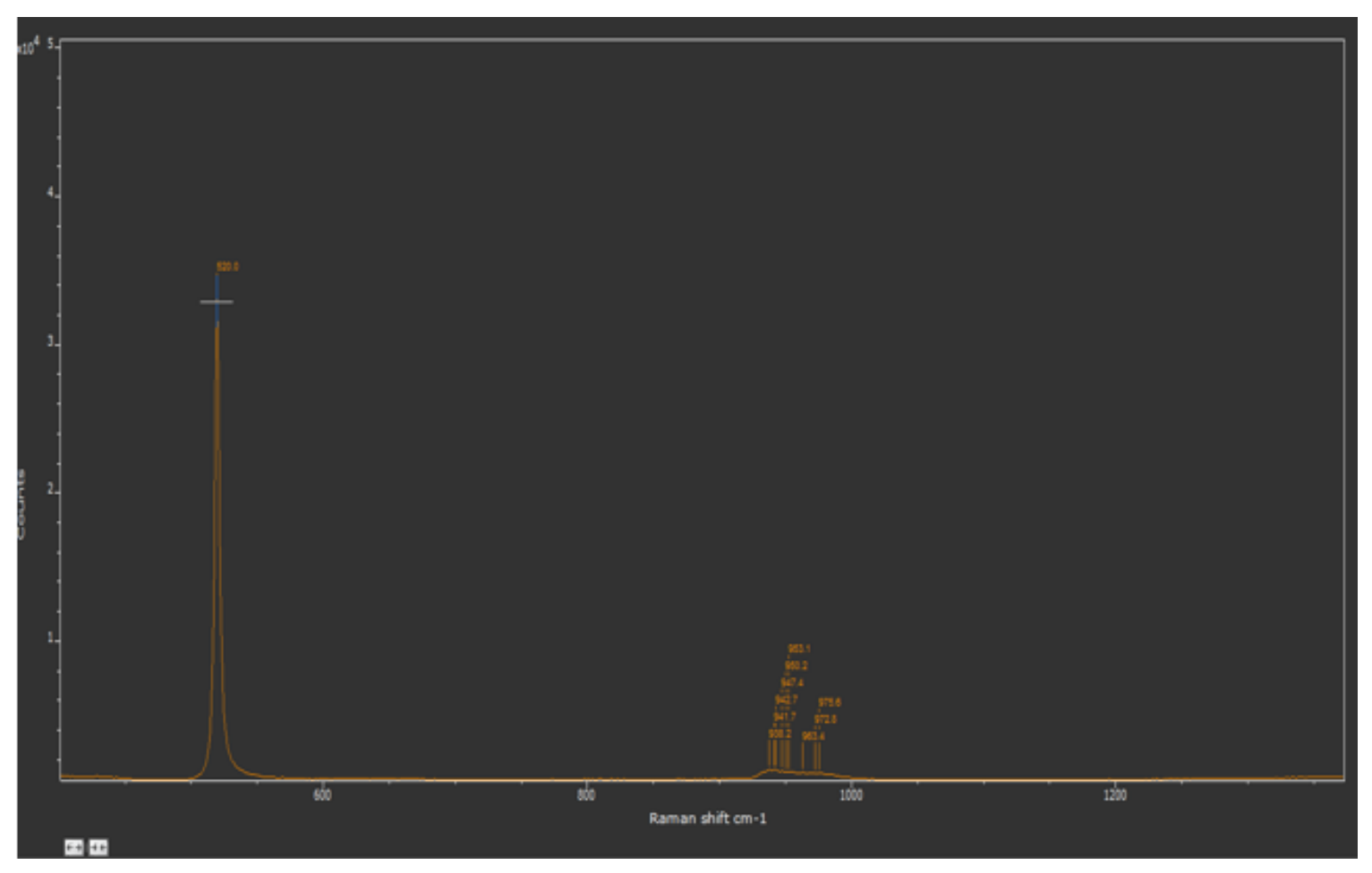

Figure 4.2: Silicon peak used as a system check

\subsection{Spectral Pre-processing}

Prior to data analysis for extracting information from Raman spectra, the data must undergo some pre-processing steps. Preprocessing is required to eliminate effects of unwanted signal, spurious artifacts and to improve signal to noise ratio. The preprocessing procedure includes: cosmic background removal, background correction, smoothing and normalization.

\subsubsection{Cosmic Ray Removal}

The first step in the preprocessing of the raw Raman spectra involves removing any cosmic ray peaks. The cosmic rays are high and narrow peaks produced by high energy particles hitting the detector. These signals show up in Raman spectra as sharp spikes, 
usually not more than 1-2 $\mathrm{cm}^{-1}$ wide compared to genuine Raman bands and they do not alter the position and intensities of the Raman peaks in the original data. Cosmic rays must be filtered out from the collected spectra and there are various mathematical methods that can be used to remove these peaks [41,42]. In this thesis all the cosmic ray peaks were removed during collection of spectra using a program written by Dean Shepherdson in Andor Basic (Appendix D).

\subsubsection{Background Subtraction/ Baseline Removal}

The untreated Raman spectra of biological samples contain the Raman signals, superimposed with a fluorescence spectrum resulting from the many organic molecules in the sample, as well as from any presence of the quartz cover slip. A crucial step in proper analysis of Raman spectra is the use of an appropriate background removal algorithm. This step separates the Raman spectrum of the cell from the broad fluorescence signal of the cell, quartz, and medium, which is often several orders of magnitude stronger than the Raman signal. Without removing the background accurately and consistently, artifacts produced by the removal may render subsequent analysis on the Raman spectrum meaningless.

Many background removal algorithms have been developed for removing florescence signal $[33,43]$. The spectrum-based method for iterative removal of fluorescence (SMIRF) algorithm developed in the MATLAB programming language by Beier et al. [44] was used for the purposes of this work. This background subtraction method utilized a fifth-order polynomial to model the fluorescence signal. This method 
takes the background spectrum as a "contaminant" spectrum that is fitted to the cell spectrum using an iterative polynomial fitting algorithm.

\subsubsection{Smoothing}

Another challenge in pre-processing is to capture important patterns in the spectra while removing noise. Baseline correction removes contributions from broad peaks or low frequency components that may exist in the Raman spectra. However the high frequency component (lower FWHM compared to Raman Peaks) of the Raman signal must also be removed.

Smoothing is a common practice used to reduce the noise potentially associated with a recorded spectrum. Information can be lost by over-smoothing the spectra, so it must be applied with great care. Various mathematical manipulation methods are available to smooth spectral data. Most smoothing algorithms rely on the fact that the Raman data vary somewhat gradually from point to point, whereas noise changes quickly [21,33]. A popular algorithm for smoothing is called Savitzky-Golay filtering. This filter is a moving-window based local polynomial fitting procedure, which requires the size of the window and polynomial order as fitting parameters. It is very important to choose an appropriate polynomial order and moving window size to retain all the important Raman peaks [45]. A Savitzky-Golay filter using $2^{\text {nd }}$-order polynomial and a window of 15 points was applied to improve the signal-to-noise ratio (SNR) in all the spectra taken for this study. 


\subsubsection{Normalization}

Following the removal of background fluorescence, unwanted overall intensity differences in Raman spectral features must be accounted for by spectral normalization. Normalization is a very important part of preprocessing, as different spectra could have been taken at different times and conditions (such as system alignment and laser power levels). There are numerous normalization techniques available in the literature. To name some of these algorithms: peak normalization, spectra area normalization and vector normalization $[33,45]$.

In peak normalization the intensity corresponding to the central frequency of a particular Raman band is used as a reference and each spectral intensity is divided by the intensity of this reference peak. For cases in which the spectra do not share a common band, it may be better to normalize the spectra so that the total area under the spectrum is unity, and all Raman intensities in a spectrum are divided by this area. Another popular normalization method is vector normalization, also called 2-norm. Mean-centered spectra are divided by the square root of the sum of the mean-centered intensities squared. In this way the sum of all intensity values squared equals one for the spectrum.

In this study individual spectra are vector-normalized for making comparisons of spectral shapes and correcting variations in spectral intensity.

\subsection{Data Analysis}

Once spectral preprocessing is carried out the data are then ready for further analysis. The data analysis typically involves data reduction and then classification, as was described in chapter 2 , section 2.9. The main interest of this work is to investigate the feasibility of 
Raman micro-spectroscopy (in vitro) to detect tumor cells that are intrinsically resistant to chemotherapy prior to the treatment, and to discriminate between two subtypes of the human ovarian carcinoma cell line A2780.

Principal Component Analysis (PCA), combined with Linear Discriminant Analysis (LDA), was performed on the processed Raman spectra with in-house software based on the R programming language. PCA was used to transform the Raman spectra from their original Raman space into an equivalent space determined by the variance in the data. The Principal Components (PC) with significant variances were selected and the PC scores corresponding to these components, along with the original class labels for the Raman spectra, were used to create a classification model with LDA. The significant principal components were selected with the restriction that the number of principal components is at most equal to the number of cells in the smallest class used in the training data to minimize over-fitting [46,47]. The first 42 principal components, accounting for $97.7 \%$ of variance were used as input to the LDA model.

Classification was performed using LDA in two steps after separating the spectra into training (75\%) and blind (25\%) sets: in the first step a classifier model comprising the various classes was developed using spectra in the training set, and the model was validated by the Leave-One-Out Cross Validation (LOOCV) method. In the second step, the LDA model created with the training set was used to classify the blind set of Raman spectra. 


\section{Chapter: Results and Discussion}

At this point, we have completed the necessary discussion of the theory, instrumentation, and computational/analytical procedure involved in this thesis. This chapter presents the results of the experiments performed with the confocal Raman micro-spectroscopy system. As explained in the last chapter, Raman spectra were acquired from two separate quartz coverslips seeded with A2780s or A2780cp cells, on the same day using the same experimental parameters. This was done in order to minimize artifacts that might inadvertently contribute to the variance in the data between the two cell types. A total of 110 spectra corresponding to the A2780s cells and 113 spectra corresponding to the A2780cp cells were acquired from four different experimental runs.

\subsection{Results}

\subsubsection{Molecular Assignment}

Figure 5.1 shows the mean of the A2780s and A2780cp vector-normalized spectra.

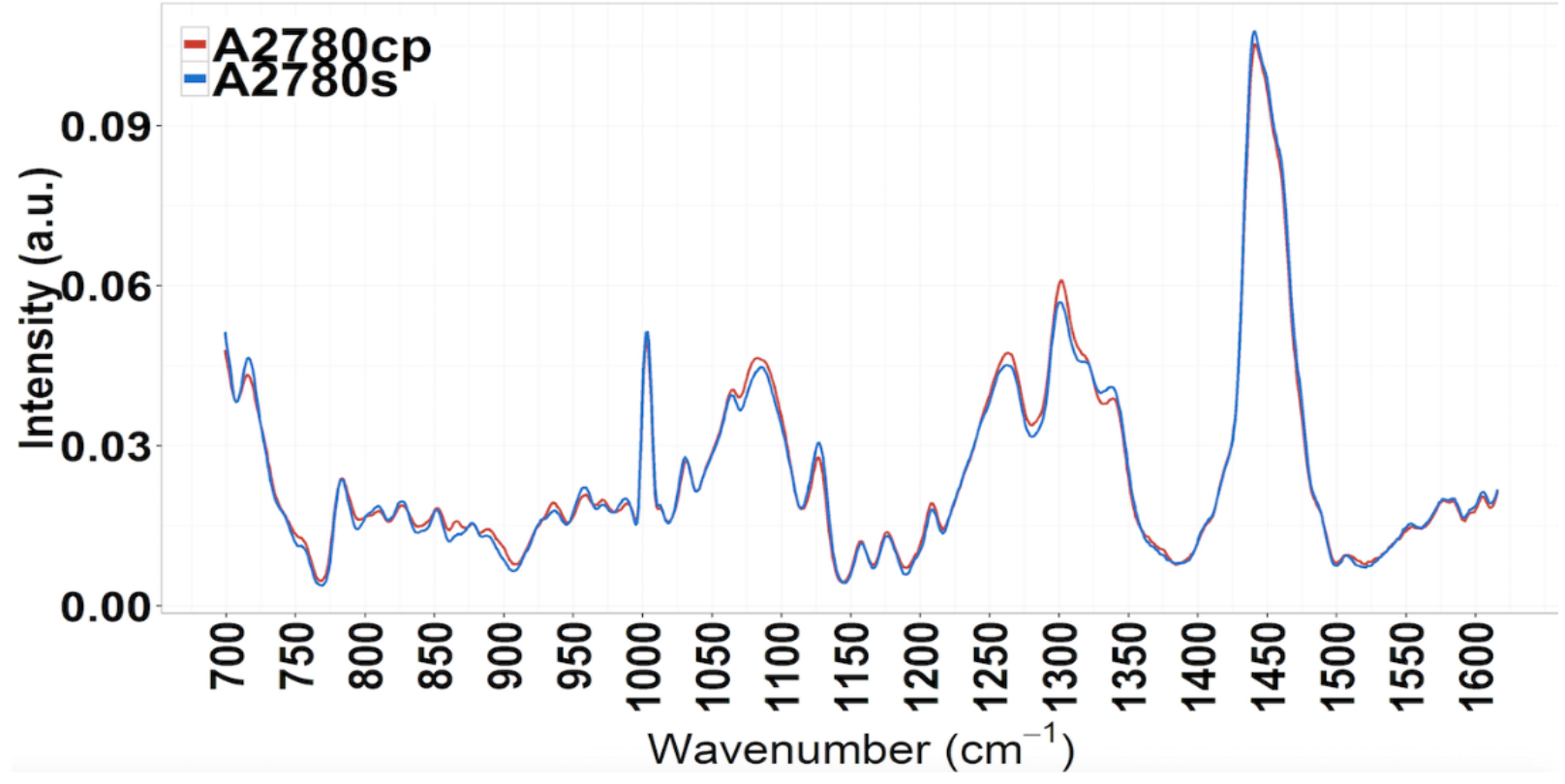

Figure 5.1: Mean Raman spectra of A2780CP (red) and A2780S (blue) cells 
The main contributions to the Raman spectra arise from vibrations of the nucleic acids, proteins, and lipids. Molecular assignments for all the major peaks are reported in table 5.1. These are in agreement with Refs [48-53] with the dominant contributions arising from the vibrations of proteins. The peaks at 746, 849, 873, 1002, 1030, 1176, 1208,1553 and $1584 \mathrm{~cm}^{-1}$ are spectral features of proteins arising from aromatic amino acids (tyrosine, phenylalanine and tryptophan). The peaks attributed to proteins that are due to the stretching or deformation of carbon atoms bonded with other nitrogen or carbon atoms are at $932,955,983,1086,1158 \mathrm{~cm}^{-1}$ while the peaks due to the vibrations of the $\mathrm{CH}_{2}$ and amide group are at 884 and 1262 and $1441 \mathrm{~cm}^{-1}$. More significantly, the peaks at 932 and $1441 \mathrm{~cm}^{-1}$ can also be attributed to the vibration of glutathione [50], a cellular agent that has been associated with resistance to cisplatin [54].

Nucleic acid features in the Raman spectrum are seen at 782, 810, 1338 and 1579 $\mathrm{cm}^{-1}$ due to vibrations of individual DNA/ RNA bases (adenine, thymine, guanine, cytosine and uracil). A comparable number of spectral features due to vibrations of lipids are seen at $718,824,1064$, and $1302 \mathrm{~cm}^{-1}$ with some overlap at $1127 \mathrm{~cm}^{-1}$ due to protein contribution. The Raman spectra for the A2780s and A2780cp cells appear almost identical, however there are some notable differences seen at 718, 932, 1086, 1127, 1262, 1301 and $1335 \mathrm{~cm}^{-1}$ attributed to the protein, nucleic acid and lipid spectral features mentioned above. 


\begin{tabular}{|c|c|}
\hline Raman Shift $\left[\mathrm{cm}^{-1}\right]$ & Assignment \\
\hline 718 & Choline (1) \\
\hline 746 & C-S trans, tryptophan (p) \\
\hline 782 & U,C,T ring breathing (d) \\
\hline 810 & O-P-O stretch RNA (d) \\
\hline 824 & O-P-O asymmetric stretch (1) \\
\hline 849 & Tyrosine ring breathing $(\mathrm{p})$ \\
\hline 862 & Phosphatic acid (1) \\
\hline 873 & Hydroxyproline, tryptophan (p) \\
\hline 884 & $\mathrm{CH}_{2}$ stretch $(\mathrm{p})$ \\
\hline 932 & Glutathione (1); C-C backbone stretch (p) \\
\hline 955 & C-C stretch (p) \\
\hline 983 & $\mathrm{C}-\mathrm{C}$ stretching $\beta$-sheet $(\mathrm{p})$ \\
\hline 1002 & Phenylalanine C-C skeletal (p) \\
\hline 1030 & Phenylalanine C-N stretch (p) \\
\hline 1064 & Skeletal C-C stretch of lipids (1) \\
\hline 1086 & C-N stretch (p) \\
\hline 1127 & C-N stretching (p); C-C stretching (l) \\
\hline 1158 & C-C; C-N stretch (p) \\
\hline 1176 & C-H bending; tyrosine, phenylalanine (p) \\
\hline 1208 & Tryptophan, phenylalanine, tyrosine (p) \\
\hline 1262 & C-H in plane bending; Amide III (p) \\
\hline
\end{tabular}




\begin{tabular}{|l|l|}
\hline 1302 & C-H deformation (l) \\
\hline 1320 & $\mathrm{G}(\mathrm{d}) ; \mathrm{C}-\mathrm{H}$ deformation (l) \\
\hline 1338 & $\mathrm{~A}, \mathrm{G}(\mathrm{d}) ; \mathrm{C}-\mathrm{H}$ deformation (p) \\
\hline 1441 & Glutathione (1); $\mathrm{CH}_{2}$ deformation (p) \\
\hline 1553 & Tryptophan (p) \\
\hline 1579 & Pyrimidine ring (d) \\
\hline 1584 & Phenylalanine (p) \\
\hline
\end{tabular}

Table 5.1: Main peaks observed in the Raman spectra of fixed A2780 cells and molecular assignments according to Refs [48-53]. Abbreviations: (d) nucleic acid, (c) carbohydrate, (p) protein, (I) lipid

\subsubsection{Classification Results}

PCA-LDA was performed on the Raman spectra after initial preprocessing as described above. The data combined from the 4 different runs for the two classes of cells were randomly split into training (75\%) and test (25\%) data sets. The first 42 PCs, accounting for $97.7 \%$ of variance were used as input to the LDA model. These PCs were selected to account for most of the variation in the data, while ensuring that class discriminatory information with smaller variance was not discarded. The empirical cumulative distribution function (CDF-emp) of the A2780s and A2780cp cells in the training set versus the LDA score is shown in figure 5.2. Also shown is the cumulative normal distribution (CDF-fit) using the mean and standard deviation of the LD score for each cell group. It is clear that there is a close match between the empirical and fitted curves. The Shapiro-Wilk test for normality gives a p-value of 0.43 for the A2780cp cells and 0.64 for the A2780s cells. This confirms that our data follows a normal distribution. 


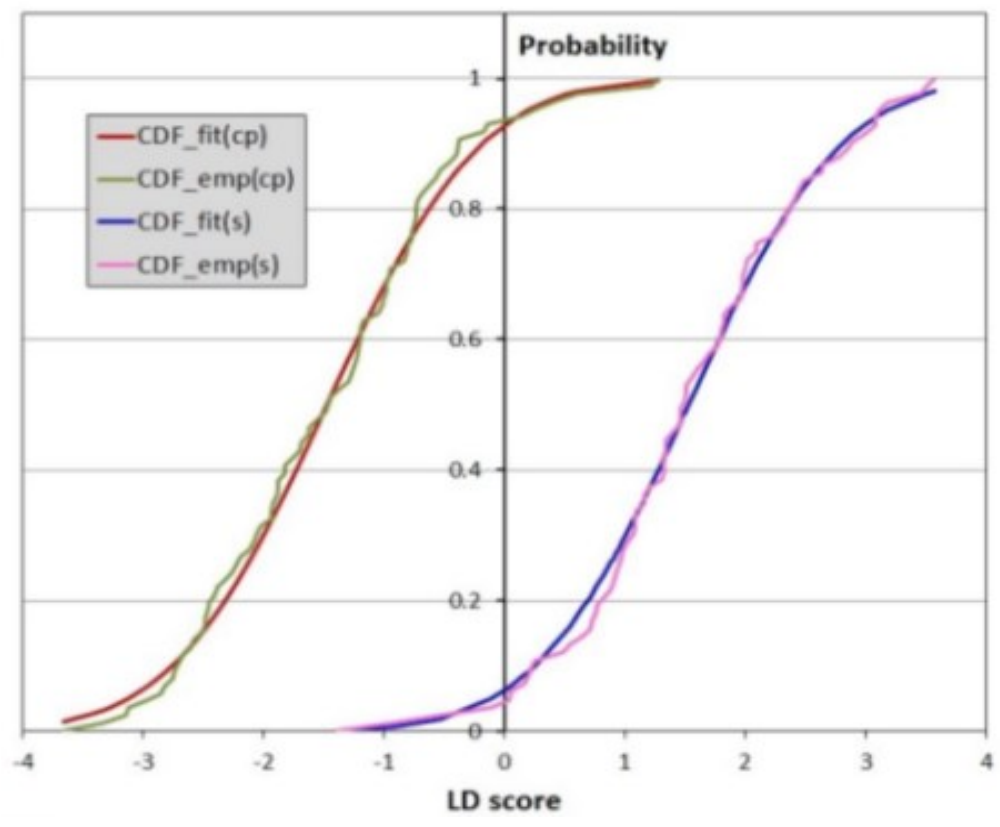

Figure 5.2: The empirical and fitted cumulative distribution function of the A2780s and A2780cp cells in the training set versus the LDA score

The histogram for the LDA scores of the A2780s and A2780cp cells in the training set is shown in figure $\mathbf{5 . 3}$ along with the fitted probability density function assuming a normal distribution based on figure 5.2. It is evident that there is good separation between the A2780s and A2780cp cells. The difference between mean LDA scores was statistically significant $(\mathrm{P}-\mathrm{value}<0.0001)$ for the classification. The histogram of LDA scores for the test data for A2780s and A2780cp cells shown at the bottom of figure $\mathbf{5 . 3}$, indicates that $\sim 88 \%$ of the test data get correctly classified. 


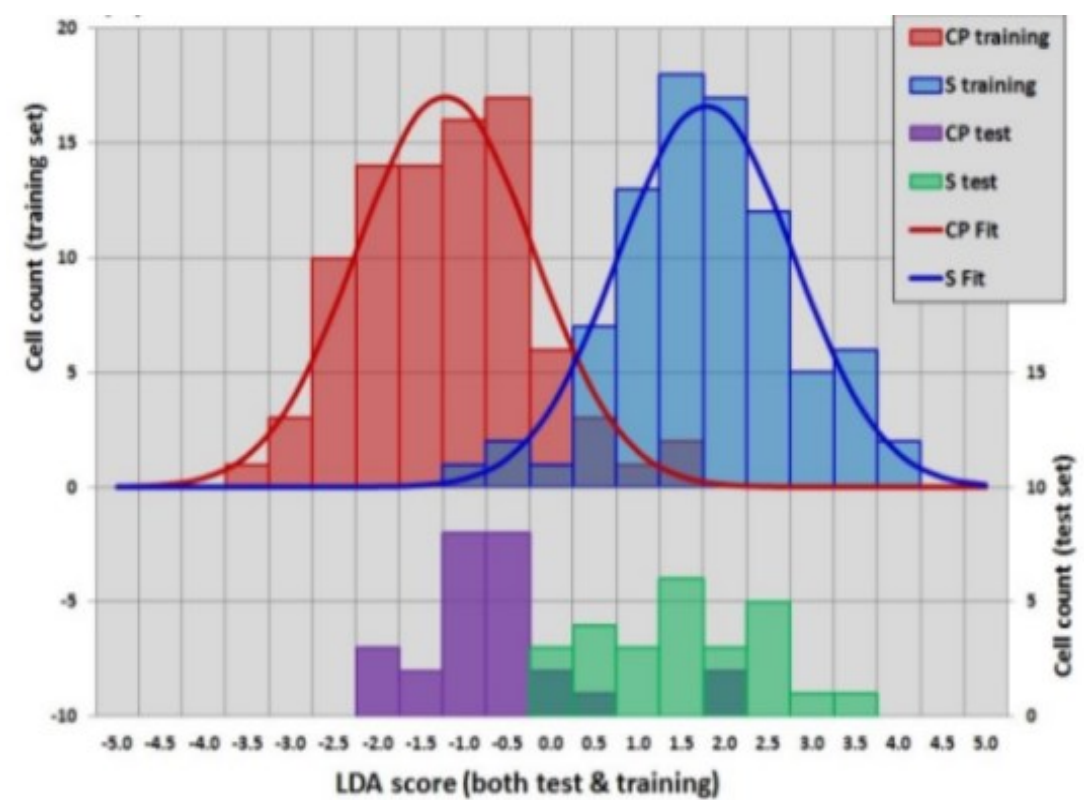

Figure 5.3: The histogram for the LDA scores of the A2780s and A2780cp cells in the training set along with the fitted probability density function derived from the normal distribution fits in figure (5.2), and at the bottom is the histogram of LDA scores for the test data for A2780s and A2780cp cells

The diagnostic classification ability of the training model was validated using two methods as described earlier. Table 5.2 shows the results for the confusion matrix for the classification of the test data as well as using LOOCV. The binary classification was performed with A2780cp as the positive cells and A2780s as negative. The classification results were used to compute the sensitivity (ratio of true positives to all classified positives), the specificity (ratio of true negatives to all classified negatives), and the accuracy (ratio of correctly classified cells to total cells). The values for the classification accuracy, sensitivity and specificity were found to be $88.5 \%$ using the test data for validation. Slightly lower values were obtained for the corresponding quantities using LOOCV and is attributed to the larger size of the test data used in this technique. 


\begin{tabular}{|r|r|c|c|c|c|c|c|}
\hline \multicolumn{2}{|c|}{ Test Data Classification Results } & \multicolumn{4}{|c|}{ LOOCV Data Classification Results } \\
\hline & Actual & & & & Actual & & \\
\hline Predicted & & A278cp & A2780s & Predicted & & A2780cp & A2780s \\
\hline & A2780cp & 23 & 3 & & A2780cp & 74 & 13 \\
\hline & A2780s & 3 & 23 & & A2780s & 17 & 67 \\
\hline & Sensitivity & $88.5 \%$ & & & Sensitivity & $81.3 \%$ & \\
\hline & Specificity & $88.5 \%$ & & & Specificity & $83.8 \%$ & \\
\hline & Accuracy & $88.5 \%$ & & & Accuracy & $82.5 \%$ & \\
\hline
\end{tabular}

Table 5.2: Confusion matrix for the test data classification and for LOOCV obtained from PCA-LDA of $A 2780 \mathrm{cp}$ and A2780s cells.

The process of randomly choosing the $75 \%$ training and $25 \%$ test data from the whole data set was repeated a total of 30 times. Similar classification results (not shown) obtained for the confusion matrix from the 30 different data sets proved that there was no bias in the choice of training and test sets used for the validation.

\subsubsection{ROC Curve Analysis}

Figure 5.4 shows the Receiver Operator Characteristics (ROC) for the detection of chemo-sensitive A2780s cells versus chemo-resistant A2780cp cells for the different possible discrimination thresholds of the classification model. It is evident that the area under the curve (AUC) of the ROC is 0.98 signifying the robustness and predictability of the classification model. 


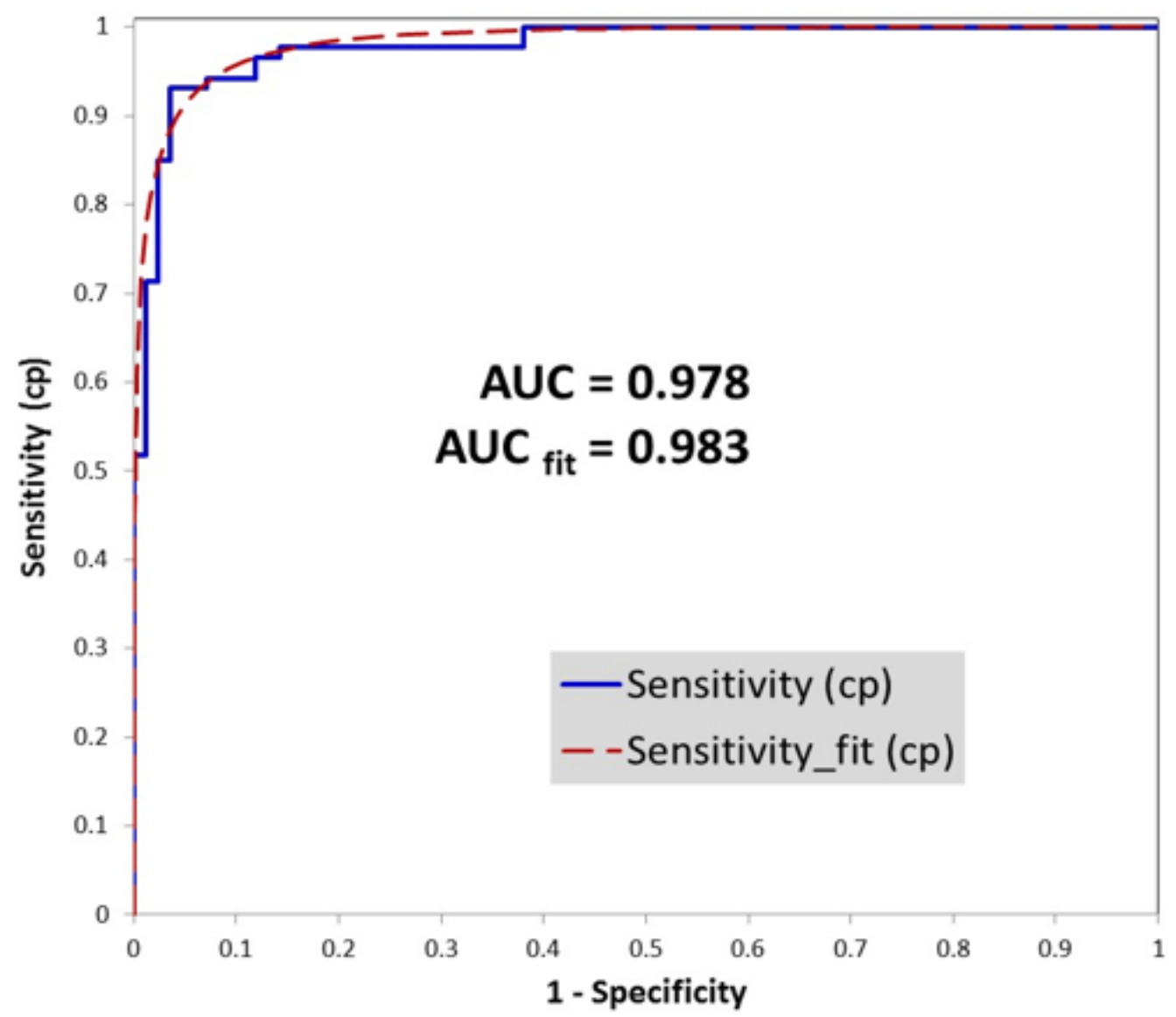

Figure 5.4: Receiver Operator Characteristics for LOOCV models (empirical and fit) of chemoresistant $\mathbf{A 2 7 8 0 c p}$ cell detection

\subsubsection{Loading Plot Analysis}

The loading plot for the Linear Discriminant (LD) function permits us to determine the major wavenumbers responsible for the discrimination $[14,47]$. The mean of the LD loading from 30 randomly chosen train-test combinations is shown in figure $\mathbf{5 . 5}$. 


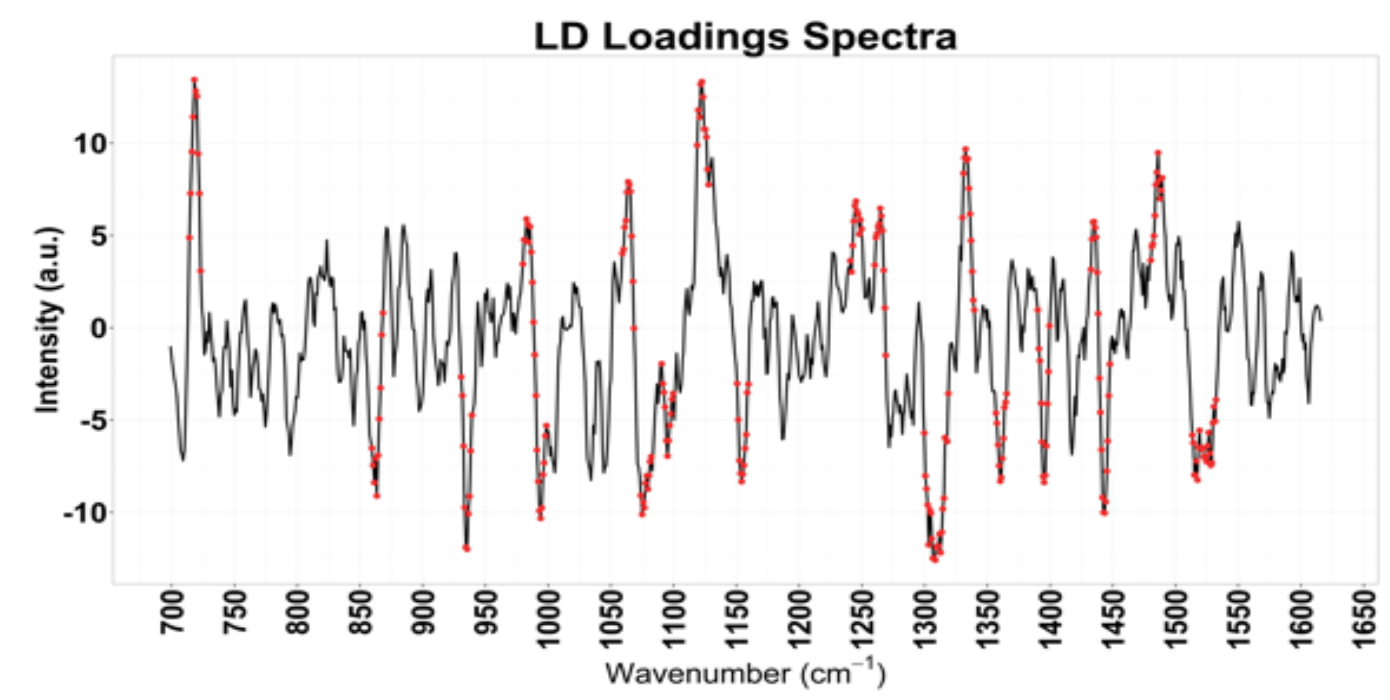

Figure 5.5: Mean LD Loading with important Raman bands indicated by red dots

The major Raman bands responsible for discrimination between A2780s and A2780cp cells are indicated in red and the corresponding molecular assignments are included in table 5.3. The major positive loadings are mainly attributed to the nucleic acids and lipids, with possible overlapping contribution from proteins. In contrast, the negative loadings are mainly due to contributions from proteins, lipids and more significantly, glutathione.

\begin{tabular}{|l|l|}
\hline Raman Shift $\left[\mathrm{cm}^{-1}\right]$ & Assignment \\
\hline Positive & \\
\hline 718 & Choline (1) \\
\hline 984 & Thymine (d) \\
\hline 1064 & Skeletal C-C stretch (1) \\
\hline 1123 & C-N stretching (p); C-C stretching (l) \\
\hline
\end{tabular}




\begin{tabular}{|c|c|}
\hline 1245 & Amide III $\beta$-sheet (p) \\
\hline 1265 & Amide III $\alpha$-helix (p); $\mathrm{CH}_{2}$ deformation (l) \\
\hline 1335 & Adenine, Guanine (d); C-H deformation (p) \\
\hline 1437 & $\mathrm{CH}_{2}$ deformation (1) \\
\hline 1485 & Adenine, Guanine (d) \\
\hline Negative & \\
\hline 864 & Phosphatic acid (l) \\
\hline 932 & Glutathione (p); C-C backbone stretch (1) \\
\hline 993 & L-Proline (p) \\
\hline 1080 & C-N stretch (p); Chain C-C stretch (l) \\
\hline 1095 & C-N stretch (p); Chain C-C stretch (1) \\
\hline 1155 & C-C \& C-N stretch (p) \\
\hline $1304-1313$ & $\mathrm{CH}_{3}, \mathrm{CH}_{2}$ twisting or bending (1) \\
\hline 1360 & Guanine (d) \\
\hline 1395 & C-H rocking (1) \\
\hline 1443 & Glutathione (p); $\mathrm{CH}_{2}$ deformation (l) \\
\hline 1518 & L-Glutamate (p) \\
\hline
\end{tabular}

Table 5.3: Tentative Raman band assignments for LD loading (figure 5.5) tabulating major peaks based on Refs [48-53]. Abbreviations: (d) nucleic acid, (c) carbohydrate, (p) protein, (l) lipid 


\subsection{Discussion}

As seen earlier in figure 5.3, the A2780cp cells spectra are negatively loaded on the LD function since the LD function separates the A2780s cells with positive scores and the A2780cp cells with negative scores. This suggests that proteins and glutathione are likely to be more abundant in the cisplatin-resistant, A2780cp cells while nucleic acids giving largely positive loadings are more likely to be abundant in the cisplatin-sensitive A2780s cells.

Earlier reports have shown that DNA repair-related proteins are over-expressed in drug-resistant ovarian carcinoma cells, and this enhances the ability of the cells to repair DNA damage [55]. Our results above indicate a higher contribution of proteins in the Raman spectra of A2780cp cells that are consistent with these previous findings. Glutathione is a cellular agent that protects cells against foreign compounds and the effects of radiation. A previous study showed that high resistance to cisplatin in human ovarian cancer cells is closely correlated with a marked increase of glutathione synthesis [54]. An interesting observation is that the peaks at 932 and $1443 \mathrm{~cm}^{-1}$ attributed to glutathione, with overlapping protein contribution in the mean Raman spectra have negative loading coefficients in the loading plot. This suggests that there is a relative increase in glutathione in the cisplatin-resistant A2780cp cells in agreement with previous findings. Genetic alterations, including frequent chromosomal losses, have been significantly associated with chemotherapy resistance in ovarian cancer [55]. Our results suggesting a likely decrease in the amount of nucleic acids in the A2780cp cells seem to be consistent with these earlier reports. 
To the best of our knowledge, there is only one previous Raman spectroscopic investigation of treatment sensitive and resistant cancer cells. In their investigation of resistant and sensitive subtypes of testicular cancer cell lines, Movasaghi et al. [51] found a significantly higher contribution of the protein band at $\sim 936 \mathrm{~cm}^{-1}$ compared to that at $884 \mathrm{~cm}^{-1}$ in the resistant subtype compared to the sensitive subtype. As seen in the loading plot in figure 5.5, the band at $\sim 935 \mathrm{~cm}^{-1}$ with the negative loading has a higher contribution than the positive loading band at $\sim 884 \mathrm{~cm}^{-1}$ in agreement with the previous work with testicular cell lines.

We next examine some factors that may improve the classification results for the discrimination to be better than $95 \%$. Our confocal Raman micro-spectroscopy setup with a laser spot size of $\sim 1 \mu \mathrm{m}$ at the sample enables us to acquire the Raman spectra from sub-cellular features in the A2780s and A2780cp cells. However, there is some uncertainty related to the reproducibility in targeting identical features in each cell of the same type for the Raman spectral acquisition. It would be possible to reduce the resulting spread in the data by increasing the laser spot size to average over the whole nucleus of each cell [57].

Another source of variance between spectra of the same cell type is the so called batch effect [58]. This is caused by systematic biological or non-biological differences between batches of cell samples from the four different runs. Better control of sample preparation and processing conditions should reduce this batch effect. Finally, application of alternative statistical techniques such as boosted trees algorithms may yield better classification since they are known to be robust to noise in the training and test data [59]. 
In order to be relevant to the clinical setting, it will be critical to demonstrate that high sensitivity and specificity for the identification of chemo-sensitivity or resistance can also be achieved in tissue. The same technique could be applied to study samples from a patient tissue bank where the samples have been retrospectively identified as chemo-sensitive or chemo-resistant. There have been a number of studies demonstrating that Raman spectroscopy can be implemented endoscopically $[3,60]$ or intraoperatively, [61] using fiber-optic probes. This approach would be highly relevant to the management of ovarian cancer patients during debulking surgery, where it could enable noninvasive in vivo prospective assessment of tumor chemo-sensitivity. 


\section{Chapter: Conclusion and Future work}

The main aim of this thesis has been the development of a confocal Raman microspectroscopy system for obtaining high resolution spectra of live and fixed cells, and ultimately to fathom the practicability of Raman spectroscopy to discriminate between platinum-sensitive and platinum-resistant ovarian tumour cells. Measurements of the human ovarian cancer cells demonstrated the ability of this instrument to acquire spectra and map the different biochemical constituent inside single cells for these two types. Concluding remarks on that work will be presented here alongside the future work required to improve the Raman system and methodology used to collect the data.

\subsection{Conclusion}

The work presented in this thesis has been motivated in part by the increasing interest to understand the biochemical differences between platinum-sensitive and platinumresistant ovarian tumour cells, and finding a reliable method to distinguish between them. This could potentially enhance current diagnostic methods. Raman micro-spectroscopy is a highly suitable technique for in vivo applications as it is non-destructive, does not require the use of external contrast enhancing agents.

In this present work, we have demonstrated the potential of Raman MicroSpectroscopy combined with multivariate analysis to differentiate between these two cell types. PCA-LDA analysis gives a diagnostic accuracy of $82 \%$ using LOOCV and $88.5 \%$ using randomized $25 \%$ test data for validation, for the discrimination between A2780s and A2780cp cells. The loading plot analysis suggests that a relative abundance in proteins and glutathione in the cisplatin-resistant cells compared to the cisplatin-sensitive 
cells is the major source of discrimination between the two types of cells. Further studies involving additional chemo-resistant and chemo-sensitive human ovarian cancer cell lines and tissue are required to evaluate and confirm these results. However, these preliminary findings support the potential application of Raman spectroscopy combined with chemometric analysis in the diagnosis of chemo-resistant disease prior to treatment.

\subsection{Future work}

In the Life Sciences it is essential to obtain detailed information about the chemical and structural composition of a sample. Routine research tasks with repetitive experiments or a large number of measurement points, as well as high-level quality control can benefit greatly from an automated instrument.

For our system, further developments are still required to automate the data collection process, so that the manual imaging time is reduced (currently $\sim 10$ minutes per cell). This would make it possible to obtain Raman measurements from more samples, while also allowing large areas to be scanned. With an automated spectral collection process, the operator would predefine the measurement routine that would be executed on the sample by the instrument. Assuming, for example, 40 cells are to be measured with a manual system, at present this would require the operator to move the stage to each of the 40 positions in order to acquire the measurements. When operating the automated system, the 40 positions would be predefined and then measured automatically without needing an operator. This can be achieved by using available software libraries provided by the manufacturer. 
In many of the lateral scans performed throughout this thesis, the sample was observed to have significant variation in thickness. In order to have consistent signal levels when building the training set, it was desirable to have a consistent depth across the scans. To obtain measurements from a constant depth, the substrate surface is first found by moving the stage so that the laser is focused. Once this is done, the stage is generally lowered by $4 \mu \mathrm{m}$ to focus on the sample. This task could also could be programmed to be executed automatically.

Further improvement to the Raman micro-spectrometer system can be achieved by using a high power laser. This would allow an additional beam path to be used by adding a second dichromic mirror. The additional path would allow the spectrometer and CCD camera to be used together, so that we do not have to constantly switch between the two devices. This would further reduce operator time by allowing snapshots and spectra to be captured from the cells, without the CCD path blocking the spectrometer.

The inherent spectral variability between cells, due to cell inhomogeneity, impedes classification accuracy. This variability can be reduced by point-scanning the whole cell or nucleus, and averaging the intensities over the collected spectra. The main disadvantage of point-scanning Raman micro-spectroscopy is that it is intrinsically slow, because a Raman spectrum is acquired from individual points within the cell.

One way to reduce spectral variability and accelerate acquisition is to alter the focusing optics so that the Raman spectrum can be collected from a larger cell area, at the expense of axial resolution. This could be achieved by changing the geometry of the laser beam using a cylindrical lens prior to the microscope objective. This will generate a line 
spot in the focal plane, so that a Raman spectrum can be obtained from an entire line, rather than a single point. This method is known as line scanning.

Another issue we have in our experiments was the systematic difference between batches (groups) of samples that were measured at different times. Batch effects may be introduced by a number of sources, such as variations in sample preparation and preservation, variations in source of samples, storage and shipment conditions, etc. In our case we attempted to minimize the batch effect between $\mathrm{s}$ and $\mathrm{cp}$ cells by acquiring data from these samples on the same day. However, to improve the analysis, the batch effect must be identified and removed between groups of the s and cp cell measurements. This batch effect must be studied with all measurements, and is not specific to s and cp cells.

Raman micro-spectroscopy is an emerging technique in cell biology, therefore there is a need to validate biochemical findings in Raman studies with an independent technique in cell biology. For the future, it would be beneficial to add wide-field fluorescence imaging capability to the existing Raman system. In this way Raman spectral images could be compared with the fluorescence images. This could also improve targeting issues since fluorescence microscopy could identify different components of the cells using different stains.

Understanding cellular structures and dynamic processes is critical in the study of cell biology. Since live cell imaging is less prone to experimental artifacts, it usually provides more reliable and relevant information than fixed cell microscopy, since fixation affects the spectral content. Our setup is equipped with a stage-top incubator and our lab has recently received a license for live cell studies. It would be beneficial to use live cells in the future. 
Data preprocessing is a crucial step in Raman data analysis to extract accurate information. There are many other data preprocessing methods which could be investigated and applied to the spectral data. In this work we used SMIRF for background removal, but it would be informative to use other available background algorithms to improve our classification results. Another spectroscopic data processing technique, which amplifies the unique Raman signatures used in classification, is the derivative of the Raman spectrum.

In this study we used established classification methods (PCA/LDA) for data analysis, but I would recommend proceeding with alternative techniques, such as logistic regression, and support vector machines (SVM), to further enhance the classification outcome.

Ultimately, in order to translate this work to the clinic, it is necessary take more samples to build a larger training set and demonstrate high accuracy in classifying blind samples. It is also necessary to develop optimized software interfaces which are automated and simplified for use by clinicians and surgical staff without the need for extensive training and compatible to the system. In the case of in vivo detection of ovarian cancer, this system must be redesigned with a fiber-optic based technique. A fiber-based probe has been designed for brain cancer by the Montreal-based company, ODS Medical, and this would be a good direction to take for ovarian cancer detection. 


\section{Appendices}

\section{Appendix A Specifications}

Specifications of the spectrometer and the automated stage have been summarized in table (A.1) and table (A.2).

\begin{tabular}{|l|l|}
\hline Focal Length (mm) & 303 \\
\hline Aperture & $\mathrm{f} / 4$ \\
\hline Focal Plane Size (mm WxH) & $28 \times 14$ \\
\hline Wavelength Resolution (nm) & 0.1 \\
\hline Wavelength Reproducibility (nm) & 0.05 \\
\hline Size (mm) & $394 \times 238 \times 208$ \\
\hline Weight (kg) & 20 \\
\hline Grating Size & $68 \times 68$ \\
\hline Wavelength Accuracy & $0.04 \mathrm{~nm}$ \\
\hline Wavelength Repeatability & $4 \mathrm{pm}$ \\
\hline Stray light & $2.2 \times 10^{-5}$ \\
\hline
\end{tabular}

Table A.1: Shamrock SR-303i Specifications [www.andor.com] 


\begin{tabular}{|l|l|}
\hline XY axis range of travel & $120 \mathrm{~mm} \times \mathbf{7 5}$ \\
$\mathbf{m m}$
\end{tabular}

FTP-2000 Linear Encoder Options

\begin{tabular}{|l|l|l|}
\hline Axis & Resolution & Scale Accuracy \\
\hline $\mathrm{XY}$ & $10 \mathrm{~nm}$ & $\pm 3 \mu \mathrm{m}$ per length of scale \\
\hline $\mathrm{Z}(12 \mathrm{~mm}$ and $25 \mathrm{~mm}$ stroke $)$ & $50 \mathrm{~nm}$ & $0.025 \mu \mathrm{m}$ per $\mathrm{mm}$ \\
\hline
\end{tabular}

Table A.2: FTP-2000 Specifications for Standard Configuration [www.asiimaging.com] 


\section{Appendix B Optical system alignment}

Accurate alignment and focusing is crucial with any optical system and could be challenging for a confocal system. Alignment is achieved by guiding the light and appropriately positioning and securing each piece of optical equipment, namely the laser, microscope, spectrometer, detector, pinhole and every mirror and lens, in the light path. The alignment and focusing of the system is completed in two parts.

Firstly, the laser is aligned into the microscope objective through a system of mirrors, and dichroic beam splitter onto a sample. The laser is aligned directly through the center of the objective, so that when the focus of the objective is varied the laser spreads in concentric circles and ideally is in focus when the sample is in focus. Dichroic beam splitter must be at the angle of $45^{\circ}$ with respect to the incoming laser. Good alignment of the laser into the microscope and onto the sample means that the maximum power available from that laser can be focused into the smallest area possible (figure B.1).

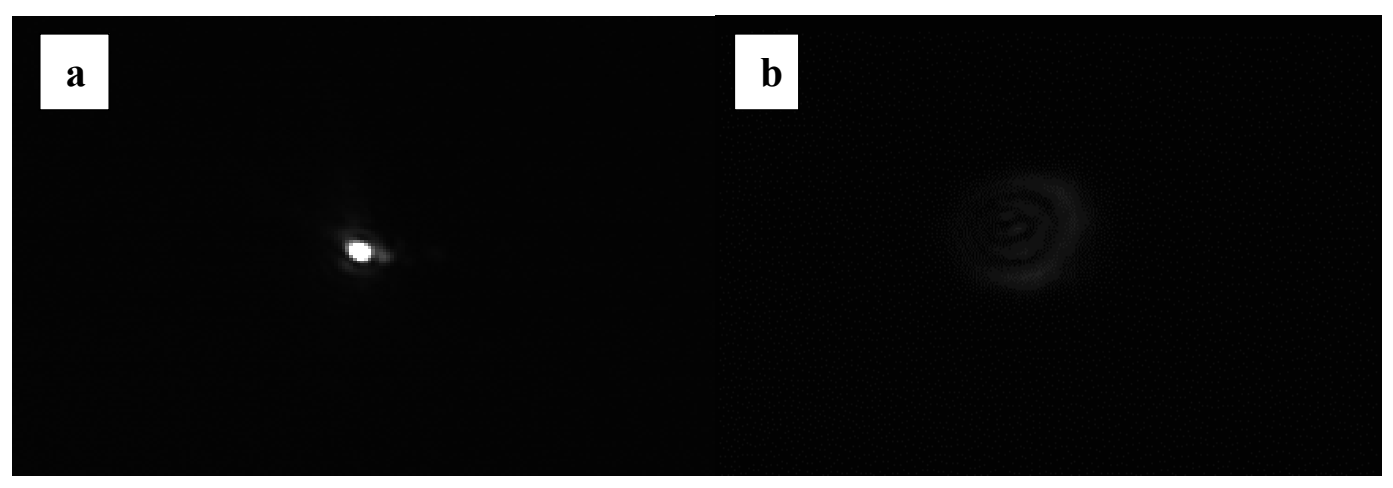

Figure B.1: Laser a) focusing and b) defocusing on the sample

This will ensure the greatest excitation of the sample and in turn strong Raman scattering. Subsequently, the resulting signal from the sample is collected. 
A flat mirror is used as the sample, as the high reflection from the mirror's surface makes the scattered light more visible for alignment. To determine the proper place of the lens located in front of the CCD camera one needs to first make sure the back scattered light is collimated. To get the proper bright-field image of the tight laser spot on the CCD camera, the lens must be adjusted.

Aligning the scattered light through the center of all the optics into the spectrometer is very challenging. The confocal pinhole must be adjusted in $\mathrm{x}-\mathrm{y}$ direction to get optimal light at the back. Proper alignment makes it possible to image the laser spot onto a few pixels of the CCD (figure B.2). The location of the image of the laser spot is also important. This image must have maximum intensity on the pixel which is located on (512x128) as shown in figure B.2 otherwise adjusting the doublet pair lens is required. Good alignment of this scattering into the spectrometer and onto the CCD is important for focusing and collecting the entire signal whilst restricting background and noise.

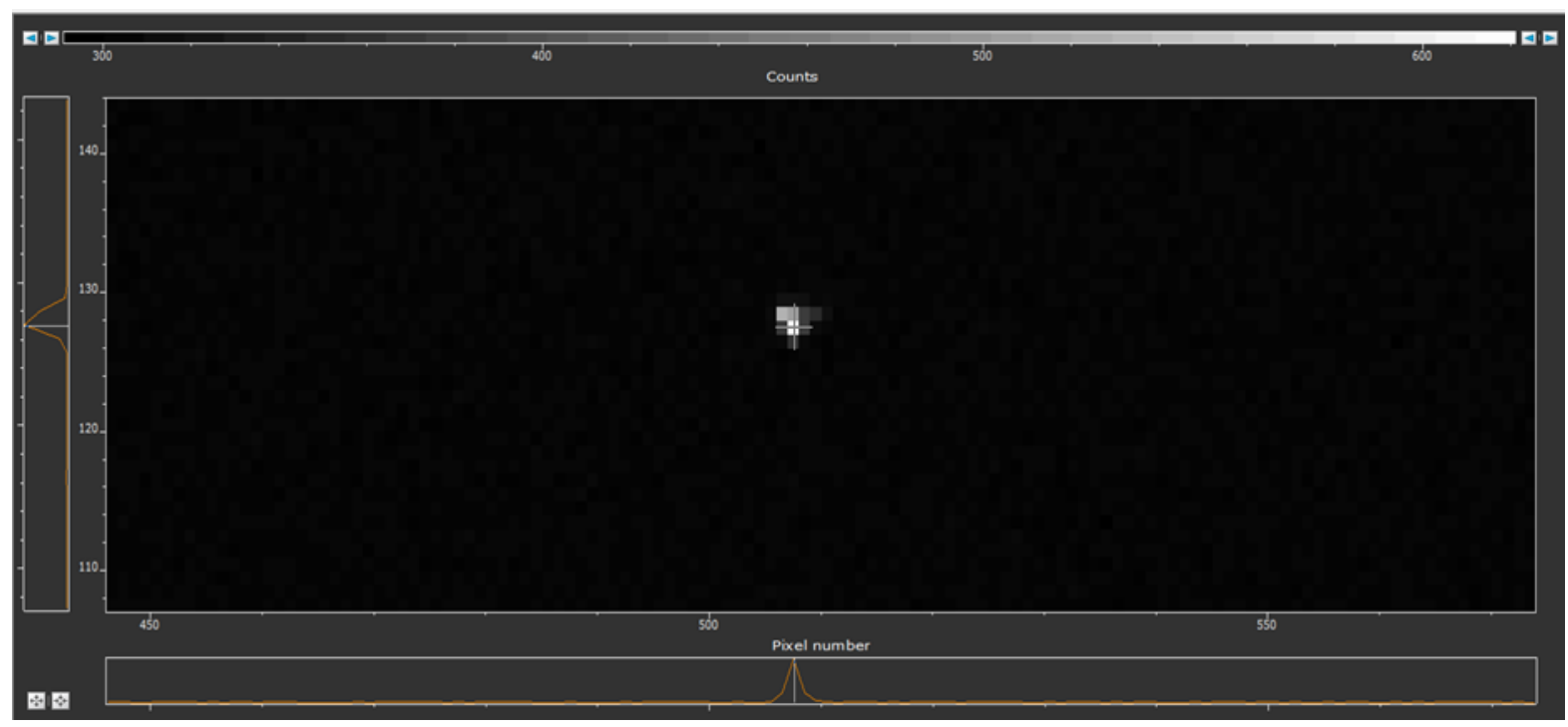

Figure B.2: Focused image on the CCD 


\section{Appendix C Instrument Calibration and Spectrum Acquisition}

In order to ensure the repeatability of the data, an instrument calibration was done before each use. The unique Raman peak of single crystal silicon located at $520 \mathrm{~cm}^{-1}$ was used as a calibration standard. The procedure that was used to operate the system and take the measurement is given below:

- Turn on the laser and let it warm up for 15 minutes.

- Turn on the Solis system and wait until the CCD temperature drops to about -85 ${ }^{0} \mathrm{C}$.

- Turn the stage console and CCD camera on.

- When laser power is stable, let laser into microscope and measure power after the objective (this should be about $34 \mathrm{~mW}$ ). Otherwise a realignment of the optics should be done.

- Focus the laser on the target (Si wafer) using the stage control to get the clearest and tightest spot size image on the CCD camera (figure B.1 a).

- Acquire a Si spectrum, setting the experimental parameters as: scan time $=1 \mathrm{sec}$, with spectral range spanning $810-935 \mathrm{~nm}$.

- If the Si peak position is shifted from $520 \mathrm{~cm}^{-1}$, we might have to change the offset for detector and grating. (offset for Detector 20 and grating 1)

- At this point the measurement system is ready for data collection from the cells. To avoid any spectral features originating from room light, the measurements should be taken in a dark room with all lights turned off.

- The laser spot must be focused on the quartz substrate, then moved onto the cells, and then the stage should be brought down from the objective by $3 \mu \mathrm{m}$. 
- Take a spectrum from each cell by taking the average of 10 exposures with an exposure time of 30 seconds for each. The spectra should be collected in the information-rich region between 700 and $1600 \mathrm{~cm}^{-1}$, with 1024 data points, and using the 1000 lines/mm grating with a $60 \mathrm{X}$ microscope objective.

- Save the images from each cell alongside the Solis output file. 


\section{Appendix D Algorithm for Cosmic Background Removal and Spectral Acqusation}

// This program is intended to make the acquisition process simple

$/ /$ and should be understood before taking data. There are some things

// you can't control through andor basic programs.

// Double Check Temperature

// You need to manually set the wavedrive control

// Check shutter settings

// Check Slit Width

// Check Grating

// CALIBRATE BEFORE TAKING DATA

$\operatorname{cls}()$

acquisitionTime $=30 / /$ Choose whatever you want

numScans $=11 / /$ total number of frames you want to take

SetAcquisitionMode(3) //Kinetics

SetReadoutMode(0) // Full Vertical Binning Mode

SetExposureTime(acquisitionTime) // Acquisition Time

SetTriggerMode(0) // Internal triggering

SetKineticCycleTime(acquisitionTime + 0.1) //allow time to clear charge on detector SetKineticNumber(numScans) // \# scans in Kinetic series

SetDataType(1) // Counts raw

print("Total time expected: "; acquisitionTime*numScans + 0.1*numScans)

SetAcquisitionType(0) // Now acquire the signal

$\operatorname{run}()$

//Ray removal

counter1 $=0$

while(counter $1<$ numScans)

rayremove2(\#0_sig $\{1\}$, \#0_sig\{counter1+1\}, 1)

counter1 = counter $1+1$

//print("Counter1: "; counter1) //error check \#of loops

wend 
//Do average on all frames except the first one

//(they should have cosmic rays removed)

// HARD CODED FOR NOW NEED TO FIGURE THIS OUT

$\# 1=\left(\left(\# 0 \_\operatorname{sig}\{2\}+\# 0 \_\operatorname{sig}\{3\}+\# 0 \_\operatorname{sig}\{4\}+\# 0 \_\operatorname{sig}\{5\}+\# 0 \_\operatorname{sig}\{6\}+\# 0 \_\operatorname{sig}\{7\}+\# 0 \_\operatorname{sig}\{8\}+\right.\right.$ $\# 0 \_$sig $\{9\}+\# 0 \_s i g\{10\}+\# 0 \_$sig $\left.\left.\{11\}\right) / 10\right) / /$ for average set

$\# 2=\# 0 \_\operatorname{sig}\{2\}+\# 0 \_\operatorname{sig}\{3\}+\# 0 \_\operatorname{sig}\{4\}+\# 0 \_\operatorname{sig}\{5\}+\# 0 \_\operatorname{sig}\{6\}+\# 0 \_\operatorname{sig}\{7\}+\# 0 \_\operatorname{sig}\{8\}+$ $\# 0 \_s i g\{9\}+\# 0 \_s i g\{10\}+\# 0 \_s i g\{11\}$

copyxcal(\#0, \#1)

copyxcal(\#0, \#2)

$/ / \# 2=\# 0 \_$sig $\{2\}+\# 0 \_s i g\{3\}+\# 0 \_s i g\{4\} / /$ for accumulated data

//copyxcal(\#0, \#2)

$/ / * * * * *$ For some reason the average keeps the $\mathrm{x}$ signals it averages...

//ie. col1 = wavenumber, $\operatorname{col} 2=$ avg, $\operatorname{col} 3-x=\# 0 \_$sig $\{2$ to $x\} \ldots$

ascii\$ = "C:\Users\SangeetaLab\Desktop\SolisOutput \"

beep()

input("Enter Sample Info", scanName\$)

$/ / \#$ temp $=\# 0 / /$ allows the file to be named by scanName\$, otherwise the \#0 overwrites it

$\#$ temp2 = \#1

SaveAsciiXY(\#temp2, ascii\$;;scanName\$;"_avg.asc", 2) // delimiter 2 = tab delimiter ----Outputs wave\# | Avg Counts | frame2 | frame 3 | frame 4.... For however many frames

$\#$ temp3 = \#2

SaveAsciiXY(\#temp3, ascii\$;scanName\$;"_acc.asc", 2) // ---- outputs wave\# |

Accumulated Counts | frame 2 | .... for however many frames

print("Run Finished")

print("Output to: "; ascii\$;scanName\$) 


\section{References}

[1] V. V. Tuchin, "Handbook of Photonics for Biomedical Science", CRC Press, Boca Raton, 2010

[2] A. Downes, A. Elfick, "Raman Spectroscopy and Related Techniques in Biomedicine", Sensors, 2010, 10, 1871-1889

[3] K. Kong, C. Kendall, N. Stone, et al., "Raman spectroscopy for medical diagnostics from in-vitro biofluid assays to in-vivo cancer detection”, Adv. Drug Deliv. Rev., $2015,89,121-134$

[4] M. B. Fenn, P. Xanthopoulos, G. Pyrgiotakis, et al., "Raman Spectroscopy for Clinical Oncology", Advances in Optical Technologies, Article ID 213783, 2011

[5] I. R. M. Ramos, A. Malkin, F. M. Lyng, "Current Advances in the Application of Raman Spectroscopy for Molecular Diagnosis of Cervical Cancer”, BioMed Research International, Article ID 561242, 2015

[6] Statistics Canada, Leading Causes of Death in Canada, Ottawa: Statistics Canada, 2015

[7] D. Holmes, L. Reading-Ikkanda, "The Problem with Platinum”, Nature, 2015, 527, S218-S219

[8] I. A. Cree, "Chemosensitivity and chemoresistance testing in ovarian cancer", Curr Opin Obstet Gynecol, 2009, 21, 39-43

[9] B. Davidson, "Recently identified drug resistance biomarkers in ovarian cancer", Expert Review of Molecular Diagnostics, 2016, 16, 569-578 
[10] J. K. Schwarz, P. W. Grigsby, F. Dehdashti, et al., "The role of 18F-FDG PET in assessing therapy response in cancer of the cervix and ovaries”, J Nucl Med, $2009,50,64 \mathrm{~S}-73 \mathrm{~S}$

[11] C. Mallidis, V. Sanchez, J. Wistuba, et al., "Raman microspectroscopy: shining a new light on reproductive medicine”, Hum Reprod Update, 2013, 0(0), 1-12

[12] C. M. Krishna, G. D. Sockalingum, L. Venteo, et al., "Evaluation of the suitability of ex vivo handled ovarian tissues for optical diagnosis by Raman microspectroscopy", Biopolymers, 2005, 79(5), 269-276

[13] K. Maheedhar, R. A. Bhat, R. Malini, et al., "Diagnosis of ovarian cancer by Raman spectroscopy: a pilot study”, Photomedicine and Laser Surgery, 2008, 26(2), 8390

[14] G. L. Owens, K. Gajjar, J. Trevisan, et al., "Vibrational biospectroscopy coupled with multivariate analysis extracts potentially diagnostic features in blood plasma/serum of ovarian cancer patients”, Journal of Biophotonics, 2014, 7(3-4), 200-209

[15] S. Borel, E. A. Prikryl, N. H. Vuong, et al., "Discrimination of normal and malignant mouse ovarian surface epithelial cells in vitro using Raman microspectroscopy”, Anal. Methods, 2015, 7, 9520-9528

[16] E. Smith, G. Dent, “Modern Raman Spectroscopy: A Practical Approach”, John Wiley \& Sons, Chichester, 2013

[17] D. J. Gardiner, P. R. Graves, "Practical Raman Spectroscopy”, Springer-Verlag, Berlin, 1989 
[18] P. Vandenabeele, "Practical Raman Spectroscopy: an introduction", John Wiley \& son, 2013

[19] I. W. Schie, T. Huser, "Methods and applications of Raman microspectroscopy to single-cell analysis", Applied Spectroscopy, 2013, 67(8), 813-828

[20] H. J. Butler, L. Ashton, B. Bird, et al., "Using Raman spectroscopy to characterize biological materials", Nature Protocols, 2016, 11(4), 664-687

[21] T. Dieing, O. Hollricher, J. Toporski, “Confocal Raman microscopy”, Springer, Berlin, 2011

[22] E. Hecht, "Optics”, $4^{\text {th }}$ Ed., Addison -Wesley, San Francisco, 2002

[23] J. M. Lerner, A. Thevenon, “The Optics of Spectroscopy: A Tutorial”, Horiba Scientific [Accessed Online: 2016]

[24] I. R. Lewis, H. G. M. Edwards, "Handbook of Raman Spectroscopy: From the Research Laboratory to the Process Line", Marcel Dekker, New York, 2001

[25] J. B. Pawley, "Handbook of Biological Confocal Microscopy", 3rd Ed., Springer, New York, 2006

[26] B. D. Beier, "Confocal Raman Microspectroscopy of Oral Streptococci”, School of Engineering and Applied Sciences University of Rochester, New York 2011

[27] G. J. Puppels, J. H. Olminkhof, G. M. Segersnolten, et al., "Laser irradiation and Raman spectroscopy of single living cells and chromosomes: sample degradation occurs with $514.5 \mathrm{~nm}$ but not with $660 \mathrm{~nm}$ laser light”, Exp Cell Res., 1991, 195(2), 361-367 
[28] I. Notingher, L. L. Hench, "Raman microspectroscopy: a noninvasive tool for studies of individual living cells in vitro", Expert Rev. Med. Devices, 2006, 3(2), 215-234

[29] L. M. Fullwood, D. Griffiths, K. Ashton, et al, "Effect of substrate choice and tissue type on tissue preparation for spectral histopathology by Raman microspectroscopy", Analyst, 2014, 139, 446-454

[30] I.T. Jolliffe, "Principal Component Analysis", Springer-Verlag, New York, 2002

[31] M. Cord, P. Cunningham, "Machine Learning Techniques for Multimedia Case Studies on Organization and Retrieval”, Springer-Verlag, Berlin, 2008

[32] J. Shlens, "A Tutorial on Principal Component Analysis: Derivation, Discussion, and Singular Value Decomposition”, 2003 [Accessed Online: 2016]

[33] R. Gautam, S. Vanga, F. Ariese, et al. "Review of multidimensional data processing approaches for Raman and infrared spectroscopy. EPJ Techniques and Instrumentation”, Springer Open, 2015, 2(1), 8

[34] A. J. Izenman, "Modern Multivariate Statistical Techniques Regression, Classification, and Manifold Learning", Springer, New York, 2008

[35] L. Rongguang, "Optical Design for Biomedical Imaging. SPIE Press Monograph Vol. PM203”, SPIE, Washington, 2011

[36] D. Grauw, C. J., Sijtsema, N. M., et al., “Axial resolution of confocal Raman microscopes: Gaussian beam theory and practice", Journal of Microscopy, 1997, 188(3), 273-279

[37] S.Wilhelm, B.Grobler, M.Gluch, et al., "Confocal Laser Scanning Microscopy Principles", ZEISS Jena, Jena, 1997 [Accessed online: 2016] 
[38] M. Niedbala, G. Alsbeih, C. E. Ng, et al. , "Equivalence of Pulsed-Dose-Rate to Low-Dose-Rate Irradiation in Tumor and Normal Cell Lines", Radiation Research, 2001, 155, 297-303

[39] A. D. Meade, C. Clarke, F. Draux, et al., "Studies of chemical fixation effects in human cell lines using Raman microspectroscopy”, Analytical and Bioanalytical Chemistry, 2010, 396(5), 1781-1791

[40] F. Draux, C. Gobinet, J. Sulé-Suso, et al., "Raman spectral imaging of single cancer cells: probing the impact of sample fixation methods", Analytical and Bioanalytical Chemistry, 2010, 397(7), 2727-2737

[41] H. G. Schulze, R. F. B. Turner, "A fast, automated, polynomial-based cosmic ray spike-removal method for the high-throughput processing of Raman spectra", Applied Spectroscopy, 2013, 67(4), 457-462

[42] H. G. Schulze, R. F. B. Turner, “A two-dimensionally coincident second difference cosmic ray spike removal method for the fully automated processing of Raman spectra”, Applied Spectroscopy, 2014, 68(2), 185-191

[43] Z. M. Zhang, S. Chen, Y. Z. Liang, "Baseline correction using adaptive iteratively reweighted penalized least squares", Analyst, 2010, 135(5), 1138-1146

[44] B. D. Beier, A. J. Berger, "Method for automated background subtraction from Raman spectra containing known contaminants", Analyst, 2009, 134(6), 1198 1202

[45] J. R. Ferraro, K. Nakamoto, "Introductory Raman Spectroscopy”, Academic Press, San Diego, 1994 
[46] P. Crow, B. Barrass, C. Kendall, et al., "The use of Raman spectroscopy to differentiate between different prostatic adenocarcinoma cell lines", British Journal of Cancer, 2005, 92(12), 2166-2170

[47] T. J. Harvey, E. Gazi,, A. Henderson, et al., "Factors influencing the discrimination and classification of prostate cancer cell lines by FTIR microspectroscopy", Analyst, 2009, 134(6), 1083

[48] Q. Matthews, A. Jirasek, J. Lum, et al., "Variability in Raman spectra of single human tumor cells cultured in vitro: correlation with cell cycle and culture confluency”, Appl. Spectroscopy, 2010, 64, 871-887

[49] S. Barkur, A. Bankapur, M. Pradhan, et al., "Probing differentiation in cancer cell lines by single-cell micro-Raman spectroscopy”, Journal of Biomedical Optics, $2015,20(8), 85001$

[50] J. D. Gelder, K. D. Gussem, P. Vandenabeele, et al., "Reference database of Raman spectra of biological molecules", Journal of Raman Spectroscopy, 2007, 38(9), $1133-1147$

[51] Z. Movasaghi, S. Rehman, I. U. Rehman, "Raman Spectroscopy of Biological Tissues”, Applied Spectroscopy Reviews, 2007, 42(5), 493-541

[52] N. Stone, C. Kendall, J. Smith, et al., "Raman spectroscopy for identification of epithelial cancers", Faraday Discussions, 2004, 126, 141-157

[53] I. Notingher, S. Verrier, S. Haque, "Spectroscopic study of human lung epithelial cells (A549) in culture: living cells versus dead cells", Biopolymers, 2003, 72(4), $230-240$ 
[54] A. K. Godwin, A. Meister, P. J. O’Dwyer, et al. "High resistance to cisplatin in human ovarian cancer cell lines is associated with marked increase of glutathione synthesis", Proceedings of the National Academy of Sciences of the United States of America, 1992, 89(7), 3070-3074

[55] F. Gong, X. Peng, Z. Zeng, et al. "Proteomic analysis of cisplatin resistance in human ovarian cancer using 2-DE method. Molecular and Cellular Biochemistry", 2011, 348(1-2), 141-147

[56] L. Osterberg, K. Levan, K. Partheen, et al., "Potential predictive markers of chemotherapy resistance in stage III ovarian serous carcinomas", BMC Cancer, $2009,9,368$

[57] I. W. Schie, L Alber, A. L. Gryshuk, et al., "Investigating drug induced changes in single, living lymphocytes based on Raman micro-spectroscopy", Analyst, 2014, 139(11), 2726-2733

[58] J. Luo, M. Schumacher, A. Scherer, et al., "A comparison of batch effect removal methods for enhancement of prediction performance using MAQC-II microarray gene expression data", The Pharmacogenomics Journal, 2010, 10(4), 278-291

[59] R. Caruana, A. Niculescu-Mizil, “An Empirical Comparison of Supervised Learning Algorithms", Proceedings of the 23rd International Conference on Machine Learning (New York), 2006, 161-168

[60] M. G. Shim, B. C. Wilson, "Development of an In Vivo Raman Spectroscopic System for Diagnostic Applications", Journal of Raman Spectroscopy, 1997, 28(2-3), 131-142 
[61] M. Jermyn, K. Mok, J. Mercier, et al., "Intraoperative brain cancer detection with Raman spectroscopy in humans", Science Translational Medicine", 2015, 7(274), $274 \mathrm{ra} 19$

[62] M Gu, T. Tannous, C. J. R. Sheppard, "Improved axial resolution in confocal fluorescence microscopy using annular pupils", Optics Communications, 1994, $110,533-539$

[63] P. J. Caspers, G. W. Lucassen, H. A. Bruining, et al., "Automated depth-scanning confocal Raman microspectrometer for rapid in vivo determination of water concentration profiles in human skin", Journal of Raman Spectroscopy, 2000, 31, $813-818$

[64] L. M. Jeremy, "Imaging Spectrometer Fundamentals for Researchers in the Bioscience - A Tutorial”, International Society for Analytical Cytology, Cytometry Part A, 2006, 69A, 712-734 